\title{
Existence of Solutions for Nonlinear Impulsive Fractional Differential Equations of Order $\alpha \in(2,3]$ with Nonlocal Boundary Conditions
}

\author{
Lihong Zhang, ${ }^{1}$ Guotao Wang, ${ }^{1}$ and Guangxing Song ${ }^{2}$ \\ ${ }^{1}$ School of Mathematics and Computer Science, Shanxi Normal University, Shanxi, Linfen 041004, China \\ ${ }^{2}$ Department of Mathematics, China University of Petroleum, Shandong, Qingdao 266555, China \\ Correspondence should be addressed to Guotao Wang, wgt2512@163.com
}

Received 2 February 2012; Revised 13 April 2012; Accepted 28 April 2012

Academic Editor: Lishan Liu

Copyright (c) 2012 Lihong Zhang et al. This is an open access article distributed under the Creative Commons Attribution License, which permits unrestricted use, distribution, and reproduction in any medium, provided the original work is properly cited.

We investigate the existence and uniqueness of solutions to the nonlocal boundary value problem for nonlinear impulsive fractional differential equations of order $\alpha \in(2,3]$. By using some wellknown fixed point theorems, sufficient conditions for the existence of solutions are established. Some examples are presented to illustrate the main results.

\section{Introduction}

Fractional differential equations arise in many engineering and scientific disciplines such as the mathematical modeling of systems and processes in the fields of physics, chemistry, aerodynamics, control theory, signal and image processing, biophysics, electrodynamics of complex medium, polymer rheology, and fitting of experimental data [1-6]. For example, one could mention the problem of anomalous diffusion [7-9], the nonlinear oscillation of earthquake can be modeled with fractional derivative [10], and fluid-dynamic traffic model with fractional derivatives [11] can eliminate the deficiency arising from the assumption to continuum traffic flow and many other $[12,13]$ recent developments in the description of anomalous transport by fractional dynamics. For some recent development on nonlinear fractional differential equations, see [14-29] and the references therein.

In this paper, we investigate a three-point boundary value problems for nonlinear impulsive fractional differential equations of order $\alpha \in(2,3]$ :

$$
\begin{gathered}
{ }^{C} D^{\alpha} u(t)=f(t, u(t)), \quad t \in J^{\prime}, \\
\Delta u^{\prime}\left(t_{k}\right)=I_{k}\left(u\left(t_{k}\right)\right), \quad k=1,2, \ldots, p,
\end{gathered}
$$




$$
\begin{gathered}
\Delta u^{\prime \prime}\left(t_{k}\right)=I_{k}^{*}\left(u\left(t_{k}\right)\right), \quad k=1,2, \ldots, p, \\
u(0)=u^{\prime}(0)=0, \quad \beta u(\eta)=u(1),
\end{gathered}
$$

where ${ }^{C} D^{\alpha}$ is the Caputo fractional derivative, $2<\alpha \leq 3, f \in C(J \times R, R), I_{k}, I_{k}^{*} \in C(R, R)$, $J=[0,1], 0=t_{0}<t_{1}<\cdots<t_{k}<\cdots<t_{p}<t_{p+1}=1, J^{\prime}=J \backslash\left\{t_{1}, t_{2}, \ldots, t_{p}\right\}, 0<\eta<1, \eta \neq t_{k}(k=$ $1,2, \ldots, p), 0<\beta<1 / \eta^{2}, \Delta u^{\prime}\left(t_{k}\right)=u^{\prime}\left(t_{k}^{+}\right)-u^{\prime}\left(t_{k}^{-}\right)$, where $u^{\prime}\left(t_{k}^{+}\right)$and $u^{\prime}\left(t_{k}^{-}\right)$denote the right and the left limits of $u^{\prime}(t)$ at $t=t_{k}(k=1,2, \ldots, p)$, respectively. $\Delta u^{\prime \prime}\left(t_{k}\right)$ has a similar meaning for $u^{\prime \prime}(t)$.

Impulsive differential equations arise in many engineering and scientific disciplines as the important mathematical modeling of systems and processes in the fields of biology, physics, engineering, and so forth. Due to their significance, it is important to study the solvability of impulsive differential equations. The theory of impulsive differential equations of integer order has emerged as an important area of investigation. Recently, the impulsive differential equations of fractional order have also attracted a considerable attention, and a variety of results can be found in the papers [30-42].

The study of multipoint boundary-value problems was initiated by Bicadze and Samarskir in [43]. Many authors since then considered nonlinear multipoint boundaryvalue problems, see [44-51] and the references therein. The multipoint boundary conditions are important in various physical problems of applied science when the controllers at the end points of the interval (under consideration) dissipate or add energy according to the sensors located at intermediate points. For example, the vibrations of a guy wire of uniform cross-section and composed of $N$ parts of different densities can be set up as a multipoint boundary-value problem.

To our knowledge, no paper has considered nonlinear impulsive fractional differential equations of order $\alpha \in(2,3]$ with nonlocal boundary conditions, that is, problem (1.1). This paper fills this gap in the literature. Our purpose here is to give the existence and uniqueness of solutions for nonlinear impulsive fractional differential equations (1.1). Our results are based on some well-known fixed point theorems.

\section{Preliminaries}

Let $J_{0}=\left[0, t_{1}\right], J_{1}=\left(t_{1}, t_{2}\right], \ldots, J_{p-1}=\left(t_{p-1}, t_{p}\right], J_{p}=\left(t_{p}, 1\right]$.

We introduce the space:

$$
P C^{2}(J, R)=\left\{u: J \longrightarrow R \mid u \in C^{2}\left(J_{k}\right), k=0,1, \ldots, p, u^{\prime}\left(t_{k}^{+}\right), u^{\prime \prime}\left(t_{k}^{+}\right) \text {exist, } k=1,2, \ldots, p,\right\},
$$

with the norm:

$$
\|u\|=\sup _{t \in J}|u(t)|, \quad\|u\|_{P C^{2}}=\max \left\{\|u\|,\left\|u^{\prime}\right\|,\left\|u^{\prime \prime}\right\|\right\} .
$$

Obviously, $P C^{2}(J, R)$ is a Banach space. 
Definition 2.1. A function $u \in P C^{2}(J, R)$ with its Caputo derivative of order $\alpha$ existing on $J$ is a solution of (1.1) if it satisfies (1.1).

Theorem 2.2 (see [52]). Let $E$ be a Banach space. Assume that $A: E \rightarrow E$ is a completely continuous operator, and the set $V=\{u \in E \mid u=\mu A u, 0<\mu<1\}$ is bounded. Then $A$ has a fixed point in $E$.

Theorem 2.3 (see [52]). Let $E$ be a Banach space. Assume that $\Omega$ is an open bounded subset of $E$ with $\theta \in \Omega$, and let $A: \bar{\Omega} \rightarrow E$ be a completely continuous operator such that

$$
\|A u\| \leq\|u\|, \quad \forall u \in \partial \Omega .
$$

Then, A has a fixed point in $\bar{\Omega}$.

Lemma 2.4. Let $2<\alpha \leq 3,1 \neq \beta \eta^{2}, \eta \in\left(t_{m}, t_{m+1}\right), m$ is a nonnegative integer, $0 \leq m \leq p$. For $a$ given $y \in C[0,1]$, a function $u$ is a solution of the following impulsive boundary value problem:

$$
\begin{gathered}
{ }^{C} D^{\alpha} u(t)=y(t), \quad t \in J^{\prime}, \\
\Delta u^{\prime}\left(t_{k}\right)=I_{k}\left(u\left(t_{k}\right)\right), \quad k=1,2, \ldots, p, \\
\Delta u^{\prime \prime}\left(t_{k}\right)=I_{k}^{*}\left(u\left(t_{k}\right)\right), \quad k=1,2, \ldots, p, \\
u(0)=u^{\prime}(0)=0, \quad \beta u(\eta)=u(1),
\end{gathered}
$$

if and only if $u$ is a solution of the impulsive fractional integral equation:

$$
u(t)=\left\{\begin{array}{ll}
\frac{1}{\Gamma(\alpha)} \int_{0}^{t}(t-s)^{\alpha-1} y(s) d s+C t^{2}, & \\
\frac{1}{\Gamma(\alpha)} \int_{t_{k}}^{t}(t-s)^{\alpha-1} y(s) d s+\frac{1}{\Gamma(\alpha)} \sum_{i=1}^{k} \int_{t_{i-1}}^{t_{i}}\left(t_{i}-s\right)^{\alpha-1} y(s) d s & \\
\quad+\sum_{i=1}^{k-1} \frac{\left(t_{k}-t_{i}\right)}{\Gamma(\alpha-1)} \int_{t_{i-1}}^{t_{i}}\left(t_{i}-s\right)^{\alpha-2} y(s) d s & \\
& +\sum_{i=1}^{k-1} \frac{\left(t_{k}-t_{i}\right)^{2}}{2 \Gamma(\alpha-2)} \int_{t_{i-1}}^{t_{i}}\left(t_{i}-s\right)^{\alpha-3} y(s) d s \\
& +\sum_{i=1}^{k} \frac{\left(t-t_{k}\right)}{\Gamma(\alpha-1)} \int_{t_{i-1}}^{t_{i}}\left(t_{i}-s\right)^{\alpha-2} y(s) d s \\
& +\sum_{i=1}^{k-1} \frac{\left(t-t_{k}\right)\left(t_{k}-t_{i}\right)}{\Gamma(\alpha-2)} \int_{t_{i-1}}^{t_{i}}\left(t_{i}-s\right)^{\alpha-3} y(s) d s \\
& +\sum_{i=1}^{k} \frac{\left(t-t_{k}\right)^{2}}{2 \Gamma(\alpha-2)} \int_{t_{i-1}}^{t_{i}}\left(t_{i}-s\right)^{\alpha-3} y(s) d s+\sum_{i=1}^{k-1}\left(t_{k}-t_{i}\right) I_{i}\left(u\left(t_{i}\right)\right) \\
& +\sum_{i=1}^{k-1} \frac{\left(t_{k}-t_{i}\right)^{2}}{2} I_{i}^{*}\left(u\left(t_{i}\right)\right)+\sum_{i=1}^{k}\left(t-t_{k}\right) I_{i}\left(u\left(t_{i}\right)\right) \\
& +\sum_{i=1}^{k-1}\left(t-t_{k}\right)\left(t_{k}-t_{i}\right) I_{i}^{*}\left(u\left(t_{i}\right)\right) \\
& +\sum_{i=1}^{k} \frac{\left(t-t_{k}\right)^{2}}{2} I_{i}^{*}\left(u\left(t_{i}\right)\right)+C t^{2},
\end{array} \quad t \in J_{k}, k=1,2, \ldots, p,\right.
$$


where

$$
\begin{aligned}
& C=\frac{1}{1-} \beta \eta^{2} \\
& \times\left\{\frac{\beta}{\Gamma(\alpha)} \int_{t_{m}}^{\eta}(\eta-s)^{\alpha-1} y(s) d s+\sum_{i=1}^{m} \frac{\beta}{\Gamma(\alpha)} \int_{t_{i-1}}^{t_{i}}\left(t_{i}-s\right)^{\alpha-1} y(s) d s\right. \\
&+\sum_{i=1}^{m-1} \frac{\beta\left(t_{m}-t_{i}\right)}{\Gamma(\alpha-1)} \int_{t_{i-1}}^{t_{i}}\left(t_{i}-s\right)^{\alpha-2} y(s) d s+\sum_{i=1}^{m-1} \frac{\beta\left(t_{m}-t_{i}\right)^{2}}{2 \Gamma(\alpha-2)} \int_{t_{i-1}}^{t_{i}}\left(t_{i}-s\right)^{\alpha-3} y(s) d s \\
&+\sum_{i=1}^{m} \frac{\beta\left(\eta-t_{m}\right)}{\Gamma(\alpha-1)} \int_{t_{i-1}}^{t_{i}}\left(t_{i}-s\right)^{\alpha-2} y(s) d s+\sum_{i=1}^{m-1} \frac{\beta\left(\eta-t_{m}\right)\left(t_{m}-t_{i}\right)}{\Gamma(\alpha-2)} \int_{t_{i-1}}^{t_{i}}\left(t_{i}-s\right)^{\alpha-3} y(s) d s \\
&+\sum_{i=1}^{m} \frac{\beta\left(\eta-t_{m}\right)^{2}}{2 \Gamma(\alpha-2)} \int_{t_{i-1}}^{t_{i}}\left(t_{i}-s\right)^{\alpha-3} y(s) d s-\frac{1}{\Gamma(\alpha)} \sum_{i=1}^{p+1} \int_{t_{i-1}}^{t_{i}}\left(t_{i}-s\right)^{\alpha-1} y(s) d s \\
&-\sum_{i=1}^{p-1} \frac{\left(t_{p}-t_{i}\right)}{\Gamma(\alpha-1)} \int_{t_{i-1}}^{t_{i}}\left(t_{i}-s\right)^{\alpha-2} y(s) d s-\sum_{i=1}^{p-1} \frac{\left(t_{p}-t_{i}\right)^{2}}{2 \Gamma(\alpha-2)} \int_{t_{i-1}}^{t_{i}}\left(t_{i}-s\right)^{\alpha-3} y(s) d s \\
&-\sum_{i=1}^{p} \frac{\left(1-t_{p}\right)}{\Gamma(\alpha-1)} \int_{t_{i-1}}^{t_{i}}\left(t_{i}-s\right)^{\alpha-2} y(s) d s-\sum_{i=1}^{p-1} \frac{\left(1-t_{p}\right)\left(t_{p}-t_{i}\right)}{\Gamma(\alpha-2)} \int_{t_{i-1}}^{t_{i}}\left(t_{i}-s\right)^{\alpha-3} y(s) d s \\
&-\sum_{i=1}^{p} \frac{\left(1-t_{p}\right)^{2}}{2 \Gamma(\alpha-2)} \int_{t_{i-1}}^{t_{i}}\left(t_{i}-s\right)^{\alpha-3} y(s) d s+\sum_{i=1}^{m-1} \beta\left(t_{m}-t_{i}\right) I_{i}\left(u\left(t_{i}\right)\right)+\sum_{i=1}^{m-1} \frac{\beta\left(t_{m}-t_{i}\right)^{2}}{2} I_{i}^{*}\left(u\left(t_{i}\right)\right) \\
&+\sum_{i=1}^{m} \beta\left(\eta-t_{m}\right) I_{i}\left(u\left(t_{i}\right)\right)+\sum_{i=1}^{m-1} \beta\left(\eta-t_{m}\right)\left(t_{m}-t_{i}\right) I_{i}^{*}\left(u\left(t_{i}\right)\right)+\sum_{i=1}^{m} \frac{\beta\left(\eta-t_{m}\right)^{2}}{2} I_{i}^{*}\left(u\left(t_{i}\right)\right) \\
&-\sum_{i=1}^{p-1}\left(t_{p}-t_{i}\right) I_{i}\left(u\left(t_{i}\right)\right)-\sum_{i=1}^{p-1} \frac{\left(t_{p}-t_{i}\right)^{2}}{2} I_{i}^{*}\left(u\left(t_{i}\right)\right)-\sum_{i=1}^{p}\left(1-t_{p}\right) I_{i}\left(u\left(t_{i}\right)\right) \\
&\left.-\sum_{i=1}^{p-1}\left(1-t_{p}\right)\left(t_{p}-t_{i}\right) I_{i}^{*}\left(u\left(t_{i}\right)\right)-\sum_{i=1}^{p} \frac{\left(1-t_{p}\right)^{2}}{2} I_{i}^{*}\left(u\left(t_{i}\right)\right)\right\} . \\
&
\end{aligned}
$$

Proof. Let $u$ is a solution of (2.4), it holds

$$
u(t)=I^{\alpha} y(t)-c_{1}-c_{2} t-c_{3} t^{2}=\frac{1}{\Gamma(\alpha)} \int_{0}^{t}(t-s)^{\alpha-1} y(s) d s-c_{1}-c_{2} t-c_{3} t^{2}, \quad t \in J_{0},
$$

for some $c_{1}, c_{2}, c_{3} \in R$. Then, we have

$$
\begin{gathered}
u^{\prime}(t)=\frac{1}{\Gamma(\alpha-1)} \int_{0}^{t}(t-s)^{\alpha-2} y(s) d s-c_{2}-2 c_{3} t, \quad t \in J_{0} \\
u^{\prime \prime}(t)=\frac{1}{\Gamma(\alpha-2)} \int_{0}^{t}(t-s)^{\alpha-3} y(s) d s-2 c_{3}, \quad t \in J_{0} .
\end{gathered}
$$

In view of $u(0)=u^{\prime}(0)=0$, it follows $c_{1}=c_{2}=0$. 
If $t \in J_{1}$, then

$$
\begin{gathered}
u(t)=\frac{1}{\Gamma(\alpha)} \int_{t_{1}}^{t}(t-s)^{\alpha-1} y(s) d s-d_{1}-d_{2}\left(t-t_{1}\right)-d_{3}\left(t-t_{1}\right)^{2}, \\
u^{\prime}(t)=\frac{1}{\Gamma(\alpha-1)} \int_{t_{1}}^{t}(t-s)^{\alpha-2} y(s) d s-d_{2}-2 d_{3}\left(t-t_{1}\right), \\
u^{\prime \prime}(t)=\frac{1}{\Gamma(\alpha-2)} \int_{t_{1}}^{t}(t-s)^{\alpha-3} y(s) d s-2 d_{3}
\end{gathered}
$$

for some $d_{1}, d_{2}, d_{3} \in R$.

Thus, we have

$$
\begin{array}{ll}
u^{\prime}\left(t_{1}^{-}\right)=\frac{1}{\Gamma(\alpha-1)} \int_{0}^{t_{1}}\left(t_{1}-s\right)^{\alpha-2} y(s) d s-2 c_{3} t_{1}, & u^{\prime}\left(t_{1}^{+}\right)=-d_{2}, \\
u^{\prime \prime}\left(t_{1}^{-}\right)=\frac{1}{\Gamma(\alpha-2)} \int_{0}^{t_{1}}\left(t_{1}-s\right)^{\alpha-3} y(s) d s-2 c_{3}, & u^{\prime \prime}\left(t_{1}^{+}\right)=-2 d_{3} .
\end{array}
$$

In view of $u\left(t_{1}^{-}\right)=u\left(t_{1}^{+}\right), \Delta u^{\prime}\left(t_{1}\right)=u^{\prime}\left(t_{1}^{+}\right)-u^{\prime}\left(t_{1}^{-}\right)=I_{1}\left(u\left(t_{1}\right)\right)$ and $\Delta u^{\prime \prime}\left(t_{1}\right)=u^{\prime \prime}\left(t_{1}^{+}\right)-$ $u^{\prime \prime}\left(t_{1}^{-}\right)=I_{1}^{*}\left(u\left(t_{1}\right)\right)$, we have

$$
\begin{gathered}
-d_{1}=\frac{1}{\Gamma(\alpha)} \int_{0}^{t_{1}}\left(t_{1}-s\right)^{\alpha-1} y(s) d s-c_{3} t_{1}^{2} \\
-d_{2}=\frac{1}{\Gamma(\alpha-1)} \int_{0}^{t_{1}}\left(t_{1}-s\right)^{\alpha-2} y(s) d s-2 c_{3} t_{1}+I_{1}\left(u\left(t_{1}\right)\right), \\
-2 d_{3}=\frac{1}{\Gamma(\alpha-2)} \int_{0}^{t_{1}}\left(t_{1}-s\right)^{\alpha-3} y(s) d s-2 c_{3}+I_{1}^{*}\left(u\left(t_{1}\right)\right) .
\end{gathered}
$$

Consequently,

$$
\begin{aligned}
u(t)= & \frac{1}{\Gamma(\alpha)} \int_{t_{1}}^{t}(t-s)^{\alpha-1} y(s) d s+\frac{1}{\Gamma(\alpha)} \int_{0}^{t_{1}}\left(t_{1}-s\right)^{\alpha-1} y(s) d s \\
& +\frac{t-t_{1}}{\Gamma(\alpha-1)} \int_{0}^{t_{1}}\left(t_{1}-s\right)^{\alpha-2} y(s) d s+\frac{\left(t-t_{1}\right)^{2}}{2 \Gamma(\alpha-2)} \int_{0}^{t_{1}}\left(t_{1}-s\right)^{\alpha-3} y(s) d s \\
& +\left(t-t_{1}\right) I_{1}\left(u\left(t_{1}\right)\right)+\frac{1}{2}\left(t-t_{1}\right)^{2} I_{1}^{*}\left(u\left(t_{1}\right)\right)-c_{3} t^{2}, \quad t \in J_{1} .
\end{aligned}
$$


Similarly, we can get

$$
\begin{aligned}
u(t)= & \frac{1}{\Gamma(\alpha)} \int_{t_{k}}^{t}(t-s)^{\alpha-1} y(s) d s+\frac{1}{\Gamma(\alpha)} \sum_{i=1}^{k} \int_{t_{i-1}}^{t_{i}}\left(t_{i}-s\right)^{\alpha-1} y(s) d s \\
& +\sum_{i=1}^{k-1} \frac{\left(t_{k}-t_{i}\right)}{\Gamma(\alpha-1)} \int_{t_{i-1}}^{t_{i}}\left(t_{i}-s\right)^{\alpha-2} y(s) d s+\sum_{i=1}^{k-1} \frac{\left(t_{k}-t_{i}\right)^{2}}{2 \Gamma(\alpha-2)} \int_{t_{i-1}}^{t_{i}}\left(t_{i}-s\right)^{\alpha-3} y(s) d s \\
& +\sum_{i=1}^{k} \frac{\left(t-t_{k}\right)}{\Gamma(\alpha-1)} \int_{t_{i-1}}^{t_{i}}\left(t_{i}-s\right)^{\alpha-2} y(s) d s+\sum_{i=1}^{k-1} \frac{\left(t-t_{k}\right)\left(t_{k}-t_{i}\right)}{\Gamma(\alpha-2)} \int_{t_{i-1}}^{t_{i}}\left(t_{i}-s\right)^{\alpha-3} y(s) d s \\
& +\sum_{i=1}^{k} \frac{\left(t-t_{k}\right)^{2}}{2 \Gamma(\alpha-2)} \int_{t_{i-1}}^{t_{i}}\left(t_{i}-s\right)^{\alpha-3} y(s) d s+\sum_{i=1}^{k-1}\left(t_{k}-t_{i}\right) I_{i}\left(u\left(t_{i}\right)\right) \\
& +\sum_{i=1}^{k-1} \frac{\left(t_{k}-t_{i}\right)^{2}}{2} I_{i}^{*}\left(u\left(t_{i}\right)\right)+\sum_{i=1}^{k}\left(t-t_{k}\right) I_{i}\left(u\left(t_{i}\right)\right)+\sum_{i=1}^{k-1}\left(t-t_{k}\right)\left(t_{k}-t_{i}\right) I_{i}^{*}\left(u\left(t_{i}\right)\right) \\
& +\sum_{i=1}^{k} \frac{\left(t-t_{k}\right)^{2}}{2} I_{i}^{*}\left(u\left(t_{i}\right)\right)-c_{3} t^{2}, \quad t \in J_{k}, k=1,2, \ldots, p .
\end{aligned}
$$

By (2.13), it follows

$$
\begin{aligned}
u(1)= & \frac{1}{\Gamma(\alpha)} \sum_{i=1}^{p+1} \int_{t_{i-1}}^{t_{i}}\left(t_{i}-s\right)^{\alpha-1} y(s) d s+\sum_{i=1}^{p-1} \frac{\left(t_{p}-t_{i}\right)}{\Gamma(\alpha-1)} \int_{t_{i-1}}^{t_{i}}\left(t_{i}-s\right)^{\alpha-2} y(s) d s \\
& +\sum_{i=1}^{p-1} \frac{\left(t_{p}-t_{i}\right)^{2}}{2 \Gamma(\alpha-2)} \int_{t_{i-1}}^{t_{i}}\left(t_{i}-s\right)^{\alpha-3} y(s) d s+\sum_{i=1}^{p} \frac{\left(1-t_{p}\right)}{\Gamma(\alpha-1)} \int_{t_{i-1}}^{t_{i}}\left(t_{i}-s\right)^{\alpha-2} y(s) d s \\
& +\sum_{i=1}^{p-1} \frac{\left(1-t_{p}\right)\left(t_{p}-t_{i}\right)}{\Gamma(\alpha-2)} \int_{t_{i-1}}^{t_{i}}\left(t_{i}-s\right)^{\alpha-3} y(s) d s+\sum_{i=1}^{p} \frac{\left(1-t_{p}\right)^{2}}{2 \Gamma(\alpha-2)} \int_{t_{i-1}}^{t_{i}}\left(t_{i}-s\right)^{\alpha-3} y(s) d s \\
& +\sum_{i=1}^{p-1}\left(t_{p}-t_{i}\right) I_{i}\left(u\left(t_{i}\right)\right)+\sum_{i=1}^{p-1} \frac{\left(t_{p}-t_{i}\right)^{2}}{2} I_{i}^{*}\left(u\left(t_{i}\right)\right)+\sum_{i=1}^{p}\left(1-t_{p}\right) I_{i}\left(u\left(t_{i}\right)\right) \\
& +\sum_{i=1}^{p-1}\left(1-t_{p}\right)\left(t_{p}-t_{i}\right) I_{i}^{*}\left(u\left(t_{i}\right)\right)+\sum_{i=1}^{p} \frac{\left(1-t_{p}\right)^{2}}{2} I_{i}^{*}\left(u\left(t_{i}\right)\right)-c_{3}, \\
u(\eta)= & \frac{1}{\Gamma(\alpha)} \int_{t_{m}}^{\eta}(\eta-s)^{\alpha-1} y(s) d s+\frac{1}{\Gamma(\alpha)} \sum_{i=1}^{m} \int_{t_{i-1}}^{t_{i}}\left(t_{i}-s\right)^{\alpha-1} y(s) d s \\
& +\sum_{i=1}^{m-1} \frac{\left(t_{m}-t_{i}\right)}{\Gamma(\alpha-1)} \int_{t_{i-1}}^{t_{i}}\left(t_{i}-s\right)^{\alpha-2} y(s) d s+\sum_{i=1}^{m-1} \frac{\left(t_{m}-t_{i}\right)^{2}}{2 \Gamma(\alpha-2)} \int_{t_{i-1}}^{t_{i}}\left(t_{i}-s\right)^{\alpha-3} y(s) d s \\
& +\sum_{i=1}^{m} \frac{\left(\eta-t_{m}\right)}{\Gamma(\alpha-1)} \int_{t_{i-1}}^{t_{i}}\left(t_{i}-s\right)^{\alpha-2} y(s) d s+\sum_{i=1}^{m-1} \frac{\left(\eta-t_{m}\right)\left(t_{m}-t_{i}\right)}{\Gamma(\alpha-2)} \int_{t_{i-1}}^{t_{i}}\left(t_{i}-s\right)^{\alpha-3} y(s) d s
\end{aligned}
$$


Abstract and Applied Analysis

$$
\begin{aligned}
& +\sum_{i=1}^{m} \frac{\left(\eta-t_{m}\right)^{2}}{2 \Gamma(\alpha-2)} \int_{t_{i-1}}^{t_{i}}\left(t_{i}-s\right)^{\alpha-3} y(s) d s+\sum_{i=1}^{m-1}\left(t_{m}-t_{i}\right) I_{i}\left(u\left(t_{i}\right)\right)+\sum_{i=1}^{m-1} \frac{\left(t_{m}-t_{i}\right)^{2}}{2} I_{i}^{*}\left(u\left(t_{i}\right)\right) \\
& +\sum_{i=1}^{m}\left(\eta-t_{m}\right) I_{i}\left(u\left(t_{i}\right)\right)+\sum_{i=1}^{m-1}\left(\eta-t_{m}\right)\left(t_{m}-t_{i}\right) I_{i}^{*}\left(u\left(t_{i}\right)\right)+\sum_{i=1}^{m} \frac{\left(\eta-t_{m}\right)^{2}}{2} I_{i}^{*}\left(u\left(t_{i}\right)\right)-c_{3} \eta^{2} .
\end{aligned}
$$

In view of the condition $\beta u(\eta)=u(1)$, we have

$$
\begin{aligned}
c_{3}=-\frac{1}{1-\beta \eta^{2}}\{ & \frac{\beta}{\Gamma(\alpha)} \int_{t_{m}}^{\eta}(\eta-s)^{\alpha-1} y(s) d s+\sum_{i=1}^{m} \frac{\beta}{\Gamma(\alpha)} \int_{t_{i-1}}^{t_{i}}\left(t_{i}-s\right)^{\alpha-1} y(s) d s \\
& +\sum_{i=1}^{m-1} \frac{\beta\left(t_{m}-t_{i}\right)}{\Gamma(\alpha-1)} \int_{t_{i-1}}^{t_{i}}\left(t_{i}-s\right)^{\alpha-2} y(s) d s+\sum_{i=1}^{m-1} \frac{\beta\left(t_{m}-t_{i}\right)^{2}}{2 \Gamma(\alpha-2)} \int_{t_{i-1}}^{t_{i}}\left(t_{i}-s\right)^{\alpha-3} y(s) d s \\
& +\sum_{i=1}^{m} \frac{\beta\left(\eta-t_{m}\right)}{\Gamma(\alpha-1)} \int_{t_{i-1}}^{t_{i}}\left(t_{i}-s\right)^{\alpha-2} y(s) d s \\
& +\sum_{i=1}^{m-1} \frac{\beta\left(\eta-t_{m}\right)\left(t_{m}-t_{i}\right)}{\Gamma(\alpha-2)} \int_{t_{i-1}}^{t_{i}}\left(t_{i}-s\right)^{\alpha-3} y(s) d s \\
& +\sum_{i=1}^{m} \frac{\beta\left(\eta-t_{m}\right)^{2}}{2 \Gamma(\alpha-2)} \int_{t_{i-1}}^{t_{i}}\left(t_{i}-s\right)^{\alpha-3} y(s) d s-\frac{1}{\Gamma(\alpha)} \sum_{i=1}^{p+1} \int_{t_{i-1}}^{t_{i}}\left(t_{i}-s\right)^{\alpha-1} y(s) d s \\
& -\sum_{i=1}^{p-1} \frac{\left(t_{p}-t_{i}\right)}{\Gamma(\alpha-1)} \int_{t_{i-1}}^{t_{i}}\left(t_{i}-s\right)^{\alpha-2} y(s) d s-\sum_{i=1}^{p-1} \frac{\left(t_{p}-t_{i}\right)^{2}}{2 \Gamma(\alpha-2)} \int_{t_{i-1}}^{t_{i}}\left(t_{i}-s\right)^{\alpha-3} y(s) d s \\
& -\sum_{i=1}^{p} \frac{\left(1-t_{p}\right)}{\Gamma(\alpha-1)} \int_{t_{i-1}}^{t_{i}}\left(t_{i}-s\right)^{\alpha-2} y(s) d s-\sum_{i=1}^{p-1} \frac{\left(1-t_{p}\right)\left(t_{p}-t_{i}\right)}{\Gamma(\alpha-2)} \int_{t_{i-1}}^{t_{i}}\left(t_{i}-s\right)^{\alpha-3} y(s) d s \\
& -\sum_{i=1}^{p} \frac{\left(1-t_{p}\right)^{2}}{2 \Gamma(\alpha-2)} \int_{t_{i-1}}^{t_{i}}\left(t_{i}-s\right)^{\alpha-3} y(s) d s+\sum_{i=1}^{m-1} \beta\left(t_{m}-t_{i}\right) I_{i}\left(u\left(t_{i}\right)\right) \\
& \left.-\sum_{i=1}^{p-1}\left(1-t_{p}\right)\left(t_{p}-t_{i}\right) I_{i}^{*}\left(u\left(t_{i}\right)\right)-\sum_{i=1}^{p} \frac{\left(1-t_{p}\right)^{2}}{2} I_{i}^{*}\left(u\left(t_{i}\right)\right)\right\} . \\
& +\sum_{i=1}^{m-1} \frac{\beta\left(t_{m}-t_{i}\right)^{2}}{2} I_{i}^{*}\left(u\left(t_{i}\right)\right) \\
& +\sum_{i=1}^{m} \beta\left(\eta-t_{m}\right) I_{i}\left(u\left(t_{i}\right)\right)+\sum_{i=1}^{m-1} \beta\left(\eta-t_{m}\right)\left(t_{m}-t_{i}\right) I_{i}^{*}\left(u\left(t_{i}\right)\right)+\sum_{i=1}^{m} \frac{\beta\left(\eta-t_{m}\right)^{2}}{2} I_{i}^{*}\left(u\left(t_{i}\right)\right) \\
& \sum_{p}-t_{i}\left(u\left(t_{i}\right)\right)-\sum_{i=1}^{p-1} \frac{\left(t_{p}-t_{i}\right)^{2}}{2} I_{i}^{*}\left(u\left(t_{i}\right)\right)-\sum_{i=1}^{p}\left(1-t_{p}\right) I_{i}\left(u\left(t_{i}\right)\right) \\
& \\
& \\
&
\end{aligned}
$$


Substituting the value of $c_{i}(i=1,2,3)$ in (2.7) and (2.13) and letting $C=-c_{3}$, we can get (2.5). Conversely, assume that $u$ is a solution of the impulsive fractional integral equation (2.5), then by a direct computation, it follows that the solution given by (2.5) satisfies (2.4).

This completes the proof.

\section{Main Results}

Let $2<\alpha \leq 3,1 \neq \beta \eta^{2}, \eta \in\left(t_{m}, t_{m+1}\right), m$ is a nonnegative integer, and $0 \leq m \leq p$. Define the operator $A: C(J) \rightarrow C(J)$ as follows:

$$
\begin{aligned}
A u(t)= & \frac{1}{\Gamma(\alpha)} \int_{t_{k}}^{t}(t-s)^{\alpha-1} f(s, u(s)) d s+\frac{1}{\Gamma(\alpha)} \sum_{i=1}^{k} \int_{t_{i-1}}^{t_{i}}\left(t_{i}-s\right)^{\alpha-1} f(s, u(s)) d s \\
& +\sum_{i=1}^{k-1} \frac{\left(t_{k}-t_{i}\right)}{\Gamma(\alpha-1)} \int_{t_{i-1}}^{t_{i}}\left(t_{i}-s\right)^{\alpha-2} f(s, u(s)) d s+\sum_{i=1}^{k-1} \frac{\left(t_{k}-t_{i}\right)^{2}}{2 \Gamma(\alpha-2)} \int_{t_{i-1}}^{t_{i}}\left(t_{i}-s\right)^{\alpha-3} f(s, u(s)) d s \\
& +\sum_{i=1}^{k} \frac{\left(t-t_{k}\right)}{\Gamma(\alpha-1)} \int_{t_{i-1}}^{t_{i}}\left(t_{i}-s\right)^{\alpha-2} f(s, u(s)) d s+\sum_{i=1}^{k-1} \frac{\left(t-t_{k}\right)\left(t_{k}-t_{i}\right)}{\Gamma(\alpha-2)} \int_{t_{i-1}}^{t_{i}}\left(t_{i}-s\right)^{\alpha-3} f(s, u(s)) d s \\
+ & \sum_{i=1}^{k} \frac{\left(t-t_{k}\right)^{2}}{2 \Gamma(\alpha-2)} \int_{t_{i-1}}^{t_{i}}\left(t_{i}-s\right)^{\alpha-3} f(s, u(s)) d s+\sum_{i=1}^{k-1}\left(t_{k}-t_{i}\right) I_{i}\left(u\left(t_{i}\right)\right)+\sum_{i=1}^{k-1} \frac{\left(t_{k}-t_{i}\right)^{2}}{2} I_{i}^{*}\left(u\left(t_{i}\right)\right) \\
+ & \sum_{i=1}^{k}\left(t-t_{k}\right) I_{i}\left(u\left(t_{i}\right)\right)+\sum_{i=1}^{k-1}\left(t-t_{k}\right)\left(t_{k}-t_{i}\right) I_{i}^{*}\left(u\left(t_{i}\right)\right)+\sum_{i=1}^{k} \frac{\left(t-t_{k}\right)^{2}}{2} I_{i}^{*}\left(u\left(t_{i}\right)\right)+\frac{t^{2}}{1-\beta \eta^{2}} \\
\times & +\frac{\beta}{\Gamma(\alpha)} \int_{t_{m}}^{\eta}(\eta-s)^{\alpha-1} f(s, u(s)) d s+\sum_{i=1}^{m} \frac{\beta}{\Gamma(\alpha)} \int_{t_{i-1}}^{t_{i}}\left(t_{i}-s\right)^{\alpha-1} f(s, u(s)) d s \\
& +\sum_{i=1}^{m-1} \frac{\beta\left(t_{m}-t_{i}\right)}{\Gamma(\alpha-1)} \int_{t_{i-1}}^{t_{i}}\left(t_{i}-s\right)^{\alpha-2} f(s, u(s)) d s \\
& +\sum_{i=1}^{m} \frac{\beta\left(\eta-t_{m}\right)^{2}}{2 \Gamma(\alpha-2)} \int_{t_{i-1}}^{t_{i}}\left(t_{i}-s\right)^{\alpha-3} f(s, u(s)) d s-\frac{1}{\Gamma(\alpha)} \sum_{i=1}^{p+1} \int_{t_{i-1}}^{t_{i}}\left(t_{i}-s\right)^{\alpha-1} f(s, u(s)) d s \\
& +\sum_{i=1}^{m-1} \frac{\beta\left(t_{m}-t_{i}\right)^{2}}{2 \Gamma(\alpha-2)} \int_{t_{i-1}}^{t_{i}}\left(t_{i}-s\right)^{\alpha-3} f(s, u(s)) d s \\
& +\sum_{i=1}^{m} \frac{\beta\left(\eta-t_{m}\right)}{\Gamma(\alpha-1)} \int_{t_{i-1}}^{t_{i}}\left(t_{i}-s\right)^{\alpha-2} f(s, u(s)) d s \\
& +\sum_{i=1}^{m-1} \frac{\beta\left(\eta-t_{m}\right)\left(t_{m}-t_{i}\right)}{\Gamma(\alpha-1)} \int_{t_{i-1}}^{t_{i}}\left(t_{i}-s\right)^{\alpha-2} f(s, u(s)) d s-\sum_{i=1}^{p-1} \frac{\left(t_{p}-t_{i}\right)^{2}}{2 \Gamma(\alpha-2)} \int_{t_{i-1}}^{t_{i}}\left(t_{i}-s\right)^{\alpha-3} f(s, u(s)) d s \\
& \\
& \\
& \\
& \\
& \\
&
\end{aligned}
$$




$$
\begin{aligned}
& -\sum_{i=1}^{p} \frac{\left(1-t_{p}\right)}{\Gamma(\alpha-1)} \int_{t_{i-1}}^{t_{i}}\left(t_{i}-s\right)^{\alpha-2} f(s, u(s)) d s \\
& -\sum_{i=1}^{p-1} \frac{\left(1-t_{p}\right)\left(t_{p}-t_{i}\right)}{\Gamma(\alpha-2)} \int_{t_{i-1}}^{t_{i}}\left(t_{i}-s\right)^{\alpha-3} f(s, u(s)) d s \\
& -\sum_{i=1}^{p} \frac{\left(1-t_{p}\right)^{2}}{2 \Gamma(\alpha-2)} \int_{t_{i-1}}^{t_{i}}\left(t_{i}-s\right)^{\alpha-3} f(s, u(s)) d s+\sum_{i=1}^{m-1} \beta\left(t_{m}-t_{i}\right) I_{i}\left(u\left(t_{i}\right)\right) \\
& +\sum_{i=1}^{m-1} \frac{\beta\left(t_{m}-t_{i}\right)^{2}}{2} I_{i}^{*}\left(u\left(t_{i}\right)\right)+\sum_{i=1}^{m} \beta\left(\eta-t_{m}\right) I_{i}\left(u\left(t_{i}\right)\right)+\sum_{i=1}^{m-1} \beta\left(\eta-t_{m}\right)\left(t_{m}-t_{i}\right) I_{i}^{*}\left(u\left(t_{i}\right)\right) \\
& +\sum_{i=1}^{m} \frac{\beta\left(\eta-t_{m}\right)^{2}}{2} I_{i}^{*}\left(u\left(t_{i}\right)\right)-\sum_{i=1}^{p-1}\left(t_{p}-t_{i}\right) I_{i}\left(u\left(t_{i}\right)\right)-\sum_{i=1}^{p-1} \frac{\left(t_{p}-t_{i}\right)^{2}}{2} I_{i}^{*}\left(u\left(t_{i}\right)\right) \\
& \left.-\sum_{i=1}^{p}\left(1-t_{p}\right) I_{i}\left(u\left(t_{i}\right)\right)-\sum_{i=1}^{p-1}\left(1-t_{p}\right)\left(t_{p}-t_{i}\right) I_{i}^{*}\left(u\left(t_{i}\right)\right)-\sum_{i=1}^{p} \frac{\left(1-t_{p}\right)^{2}}{2} I_{i}^{*}\left(u\left(t_{i}\right)\right)\right\}
\end{aligned}
$$

then (1.1) has a solution if and only if the operator $A$ has a fixed point.

Lemma 3.1. The operator $A: C(J) \rightarrow C(J)$ is completely continuous.

Proof. Obviously, $A$ is continuous in view of continuity of $f, I_{k}$, and $I_{k}^{*}$.

Let $\Omega \subset C(J)$ be bounded. Then, there exist positive constants $L_{i}>0(i=1,2,3)$ such that $|f(t, u)| \leq L_{1},\left|I_{k}(u)\right| \leq L_{2}$ and $\left|I_{k}^{*}(u)\right| \leq L_{3}$, for all $u \in \Omega$. Thus, for all $u \in \Omega$, we have

$|(A u)(t)|$

$$
\begin{aligned}
\leq & \frac{1}{\Gamma(\alpha)} \int_{t_{k}}^{t}(t-s)^{\alpha-1}|f(s, u(s))| d s+\frac{1}{\Gamma(\alpha)} \sum_{i=1}^{k} \int_{t_{i-1}}^{t_{i}}\left(t_{i}-s\right)^{\alpha-1}|f(s, u(s))| d s \\
& +\sum_{i=1}^{k-1} \frac{\left(t_{k}-t_{i}\right)}{\Gamma(\alpha-1)} \int_{t_{i-1}}^{t_{i}}\left(t_{i}-s\right)^{\alpha-2}|f(s, u(s))| d s+\sum_{i=1}^{k-1} \frac{\left(t_{k}-t_{i}\right)^{2}}{2 \Gamma(\alpha-2)} \int_{t_{i-1}}^{t_{i}}\left(t_{i}-s\right)^{\alpha-3}|f(s, u(s))| d s \\
& +\sum_{i=1}^{k} \frac{\left(t-t_{k}\right)}{\Gamma(\alpha-1)} \int_{t_{i-1}}^{t_{i}}\left(t_{i}-s\right)^{\alpha-2}|f(s, u(s))| d s+\sum_{i=1}^{k-1} \frac{\left(t-t_{k}\right)\left(t_{k}-t_{i}\right)}{\Gamma(\alpha-2)} \int_{t_{i-1}}^{t_{i}}\left(t_{i}-s\right)^{\alpha-3}|f(s, u(s))| d s \\
& +\sum_{i=1}^{k} \frac{\left(t-t_{k}\right)^{2}}{2 \Gamma(\alpha-2)} \int_{t_{i-1}}^{t_{i}}\left(t_{i}-s\right)^{\alpha-3}|f(s, u(s))| d s+\sum_{i=1}^{k-1}\left(t_{k}-t_{i}\right)\left|I_{i}\left(u\left(t_{i}\right)\right)\right|+\sum_{i=1}^{k-1} \frac{\left(t_{k}-t_{i}\right)^{2}}{2}\left|I_{i}^{*}\left(u\left(t_{i}\right)\right)\right| \\
& +\sum_{i=1}^{k}\left(t-t_{k}\right)\left|I_{i}\left(u\left(t_{i}\right)\right)\right|+\sum_{i=1}^{k-1}\left(t-t_{k}\right)\left(t_{k}-t_{i}\right)\left|I_{i}^{*}\left(u\left(t_{i}\right)\right)\right|+\sum_{i=1}^{k} \frac{\left(t-t_{k}\right)^{2}}{2}\left|I_{i}^{*}\left(u\left(t_{i}\right)\right)\right|+\frac{t^{2}}{1-\beta \eta^{2}} \\
& \times\left\{\frac{\beta}{\Gamma(\alpha)} \int_{t_{m}}^{\eta}(\eta-s)^{\alpha-1}|f(s, u(s))| d s+\sum_{i=1}^{m} \frac{\beta}{\Gamma(\alpha)} \int_{t_{i-1}}^{t_{i}}\left(t_{i}-s\right)^{\alpha-1}|f(s, u(s))| d s\right. \\
& +\sum_{i=1}^{m-1} \frac{\beta\left(t_{m}-t_{i}\right)}{\Gamma(\alpha-1)} \int_{t_{i-1}}^{t_{i}}\left(t_{i}-s\right)^{\alpha-2}|f(s, u(s))| d s
\end{aligned}
$$




$$
\begin{aligned}
& +\sum_{i=1}^{m-1} \frac{\beta\left(t_{m}-t_{i}\right)^{2}}{2 \Gamma(\alpha-2)} \int_{t_{i-1}}^{t_{i}}\left(t_{i}-s\right)^{\alpha-3}|f(s, u(s))| d s \\
& +\sum_{i=1}^{m} \frac{\beta\left(\eta-t_{m}\right)}{\Gamma(\alpha-1)} \int_{t_{i-1}}^{t_{i}}\left(t_{i}-s\right)^{\alpha-2}|f(s, u(s))| d s \\
& +\sum_{i=1}^{m-1} \frac{\beta\left(\eta-t_{m}\right)\left(t_{m}-t_{i}\right)}{\Gamma(\alpha-2)} \int_{t_{i-1}}^{t_{i}}\left(t_{i}-s\right)^{\alpha-3}|f(s, u(s))| d s \\
& +\sum_{i=1}^{m} \frac{\beta\left(\eta-t_{m}\right)^{2}}{2 \Gamma(\alpha-2)} \int_{t_{i-1}}^{t_{i}}\left(t_{i}-s\right)^{\alpha-3}|f(s, u(s))| d s+\frac{1}{\Gamma(\alpha)} \sum_{i=1}^{p+1} \int_{t_{i-1}}^{t_{i}}\left(t_{i}-s\right)^{\alpha-1}|f(s, u(s))| d s \\
& +\sum_{i=1}^{p-1} \frac{\left(t_{p}-t_{i}\right)}{\Gamma(\alpha-1)} \int_{t_{i-1}}^{t_{i}}\left(t_{i}-s\right)^{\alpha-2}|f(s, u(s))| d s+\sum_{i=1}^{p-1} \frac{\left(t_{p}-t_{i}\right)^{2}}{2 \Gamma(\alpha-2)} \int_{t_{i-1}}^{t_{i}}\left(t_{i}-s\right)^{\alpha-3}|f(s, u(s))| d s \\
& +\sum_{i=1}^{p} \frac{\left(1-t_{p}\right)}{\Gamma(\alpha-1)} \int_{t_{i-1}}^{t_{i}}\left(t_{i}-s\right)^{\alpha-2}|f(s, u(s))| d s \\
& +\sum_{i=1}^{p-1} \frac{\left(1-t_{p}\right)\left(t_{p}-t_{i}\right)}{\Gamma(\alpha-2)} \int_{t_{i-1}}^{t_{i}}\left(t_{i}-s\right)^{\alpha-3}|f(s, u(s))| d s \\
& +\sum_{i=1}^{p} \frac{\left(1-t_{p}\right)^{2}}{2 \Gamma(\alpha-2)} \int_{t_{i-1}}^{t_{i}}\left(t_{i}-s\right)^{\alpha-3}|f(s, u(s))| d s+\sum_{i=1}^{m-1} \beta\left(t_{m}-t_{i}\right)\left|I_{i}\left(u\left(t_{i}\right)\right)\right| \\
& +\sum_{i=1}^{m-1} \frac{\beta\left(t_{m}-t_{i}\right)^{2}}{2}\left|I_{i}^{*}\left(u\left(t_{i}\right)\right)\right|+\sum_{i=1}^{m} \beta\left(\eta-t_{m}\right)\left|I_{i}\left(u\left(t_{i}\right)\right)\right|+\sum_{i=1}^{m-1} \beta\left(\eta-t_{m}\right)\left(t_{m}-t_{i}\right)\left|I_{i}^{*}\left(u\left(t_{i}\right)\right)\right| \\
& +\sum_{i=1}^{m} \frac{\beta\left(\eta-t_{m}\right)^{2}}{2}\left|I_{i}^{*}\left(u\left(t_{i}\right)\right)\right|+\sum_{i=1}^{p-1}\left(t_{p}-t_{i}\right)\left|I_{i}\left(u\left(t_{i}\right)\right)\right|+\sum_{i=1}^{p-1} \frac{\left(t_{p}-t_{i}\right)^{2}}{2}\left|I_{i}^{*}\left(u\left(t_{i}\right)\right)\right| \\
& \left.+\sum_{i=1}^{p}\left(1-t_{p}\right)\left|I_{i}\left(u\left(t_{i}\right)\right)\right|+\sum_{i=1}^{p-1}\left(1-t_{p}\right)\left(t_{p}-t_{i}\right)\left|I_{i}^{*}\left(u\left(t_{i}\right)\right)\right|+\sum_{i=1}^{p} \frac{\left(1-t_{p}\right)^{2}}{2}\left|I_{i}^{*}\left(u\left(t_{i}\right)\right)\right|\right\} \\
& \leq \frac{L_{1}}{\Gamma(\alpha)} \int_{t_{k}}^{t}(t-s)^{\alpha-1} d s+\frac{L_{1}}{\Gamma(\alpha)} \sum_{i=1}^{p} \int_{t_{i-1}}^{t_{i}}\left(t_{i}-s\right)^{\alpha-1} d s+\sum_{i=1}^{p-1} \frac{L_{1}}{\Gamma(\alpha-1)} \int_{t_{i-1}}^{t_{i}}\left(t_{i}-s\right)^{\alpha-2} d s \\
& +\sum_{i=1}^{p-1} \frac{L_{1}}{2 \Gamma(\alpha-2)} \int_{t_{i-1}}^{t_{i}}\left(t_{i}-s\right)^{\alpha-3} d s+\sum_{i=1}^{p} \frac{L_{1}}{\Gamma(\alpha-1)} \int_{t_{i-1}}^{t_{i}}\left(t_{i}-s\right)^{\alpha-2} d s \\
& +\sum_{i=1}^{p-1} \frac{L_{1}}{\Gamma(\alpha-2)} \int_{t_{i-1}}^{t_{i}}\left(t_{i}-s\right)^{\alpha-3} d s+\sum_{i=1}^{p} \frac{L_{1}}{2 \Gamma(\alpha-2)} \int_{t_{i-1}}^{t_{i}}\left(t_{i}-s\right)^{\alpha-3} d s+\sum_{i=1}^{p-1} L_{2}+\sum_{i=1}^{p-1} \frac{1}{2} L_{3} \\
& +\sum_{i=1}^{p} L_{2}+\sum_{i=1}^{p-1} L_{3}+\sum_{i=1}^{p} \frac{1}{2} L_{3}+\frac{1}{1-\beta \eta^{2}} \\
& \times\left\{\frac{L_{1} \beta}{\Gamma(\alpha)} \int_{t_{m}}^{\eta}(\eta-s)^{\alpha-1} d s+\sum_{i=1}^{p} \frac{L_{1} \beta}{\Gamma(\alpha)} \int_{t_{i-1}}^{t_{i}}\left(t_{i}-s\right)^{\alpha-1} d s\right.
\end{aligned}
$$




$$
\begin{aligned}
& +\sum_{i=1}^{p-1} \frac{L_{1} \beta}{\Gamma(\alpha-1)} \int_{t_{i-1}}^{t_{i}}\left(t_{i}-s\right)^{\alpha-2} d s+\sum_{i=1}^{p-1} \frac{L_{1} \beta}{2 \Gamma(\alpha-2)} \int_{t_{i-1}}^{t_{i}}\left(t_{i}-s\right)^{\alpha-3} d s \\
& +\sum_{i=1}^{p} \frac{L_{1} \beta}{\Gamma(\alpha-1)} \int_{t_{i-1}}^{t_{i}}\left(t_{i}-s\right)^{\alpha-2} d s+\sum_{i=1}^{p-1} \frac{L_{1} \beta}{\Gamma(\alpha-2)} \int_{t_{i-1}}^{t_{i}}\left(t_{i}-s\right)^{\alpha-3} d s \\
& +\sum_{i=1}^{p} \frac{L_{1} \beta}{2 \Gamma(\alpha-2)} \int_{t_{i-1}}^{t_{i}}\left(t_{i}-s\right)^{\alpha-3} d s+\sum_{i=1}^{p+1} \frac{L_{1}}{\Gamma(\alpha)} \int_{t_{i-1}}^{t_{i}}\left(t_{i}-s\right)^{\alpha-1} d s \\
& +\sum_{i=1}^{p-1} \frac{L_{1}}{\Gamma(\alpha-1)} \int_{t_{i-1}}^{t_{i}}\left(t_{i}-s\right)^{\alpha-2} d s+\sum_{i=1}^{p-1} \frac{L_{1}}{2 \Gamma(\alpha-2)} \int_{t_{i-1}}^{t_{i}}\left(t_{i}-s\right)^{\alpha-3} d s \\
& +\sum_{i=1}^{p} \frac{L_{1}}{\Gamma(\alpha-1)} \int_{t_{i-1}}^{t_{i}}\left(t_{i}-s\right)^{\alpha-2} d s+\sum_{i=1}^{p-1} \frac{L_{1}}{\Gamma(\alpha-2)} \int_{t_{i-1}}^{t_{i}}\left(t_{i}-s\right)^{\alpha-3} d s \\
& +\sum_{i=1}^{p} \frac{L_{1}}{2 \Gamma(\alpha-2)} \int_{t_{i-1}}^{t_{i}}\left(t_{i}-s\right)^{\alpha-3} d s+\sum_{i=1}^{p-1} \beta L_{2}+\sum_{i=1}^{p-1} \frac{\beta}{2} L_{3}+\sum_{i=1}^{p} \beta L_{2}+\sum_{i=1}^{p-1} \beta L_{3} \\
& \left.+\sum_{i=1}^{p} \frac{\beta}{2} L_{3}+\sum_{i=1}^{p-1} L_{2}+\sum_{i=1}^{p-1} \frac{1}{2} L_{3}+\sum_{i=1}^{p} L_{2}+\sum_{i=1}^{p-1} L_{3}+\sum_{i=1}^{p} \frac{1}{2} L_{3}\right\} \\
& \leq \frac{L_{1}}{\Gamma(\alpha+1)}+\frac{p L_{1}}{\Gamma(\alpha+1)}+\frac{(p-1) L_{1}}{\Gamma(\alpha)}+\frac{(p-1) L_{1}}{2 \Gamma(\alpha-1)}+\frac{p L_{1}}{\Gamma(\alpha)}+\frac{(p-1) L_{1}}{\Gamma(\alpha-1)}+\frac{p L_{1}}{2 \Gamma(\alpha-1)} \\
& +(p-1) L_{2}+\frac{p-1}{2} L_{3}+p L_{2}+(p-1) L_{3}+\frac{p}{2} L_{3}+\frac{1}{1-\beta \eta^{2}} \\
& \times\left\{\frac{\beta L_{1}}{\Gamma(\alpha+1)}+\frac{\beta p L_{1}}{\Gamma(\alpha+1)}+\frac{\beta(p-1) L_{1}}{\Gamma(\alpha)}+\frac{\beta(p-1) L_{1}}{2 \Gamma(\alpha-1)}+\frac{\beta p L_{1}}{\Gamma(\alpha)}+\frac{\beta(p-1) L_{1}}{\Gamma(\alpha-1)}+\frac{\beta p L_{1}}{2 \Gamma(\alpha-1)}\right. \\
& +\frac{(p+1) L_{1}}{\Gamma(\alpha+1)}+\frac{(p-1) L_{1}}{\Gamma(\alpha)}+\frac{(p-1) L_{1}}{2 \Gamma(\alpha-1)}+\frac{p L_{1}}{\Gamma(\alpha)} \\
& +\frac{(p-1) L_{1}}{\Gamma(\alpha-1)}+\frac{p L_{1}}{2 \Gamma(\alpha-1)}+\beta(p-1) L_{2}+\frac{\beta(p-1)}{2} L_{3} \\
& \left.+\beta p L_{2}+\beta(p-1) L_{3}+\frac{\beta p}{2} L_{3}+(p-1) L_{2}+\frac{p-1}{2} L_{3}+p L_{2}+(p-1) L_{3}+\frac{p}{2} L_{3}\right\} \\
& =\frac{2+\beta\left(1-\eta^{2}\right)}{1-\beta \eta^{2}}\left\{\frac{(p+1) L_{1}}{\Gamma(\alpha+1)}+\frac{(2 p-1) L_{1}}{\Gamma(\alpha)}+\frac{(4 p-3) L_{1}}{2 \Gamma(\alpha-1)}+(2 p-1) L_{2}+\left(2 p-\frac{3}{2}\right) L_{3}\right\} \text {, }
\end{aligned}
$$

which implies

$$
\begin{aligned}
\|A u\| \leq & \frac{2+\beta\left(1-\eta^{2}\right)}{1-\beta \eta^{2}} \\
& \times\left\{\frac{(p+1) L_{1}}{\Gamma(\alpha+1)}+\frac{(2 p-1) L_{1}}{\Gamma(\alpha)}+\frac{(4 p-3) L_{1}}{2 \Gamma(\alpha-1)}+(2 p-1) L_{2}+\left(2 p-\frac{3}{2}\right) L_{3}\right\}:=L .
\end{aligned}
$$


On the other hand, for any $t \in J_{k}, 0 \leq k \leq p$, we have

$$
\begin{aligned}
& \left|(A u)^{\prime}(t)\right| \leq \frac{1}{\Gamma(\alpha-1)} \int_{t_{k}}^{t}(t-s)^{\alpha-2}|f(s, u(s))| d s+\sum_{i=1}^{k} \frac{1}{\Gamma(\alpha-1)} \int_{t_{i-1}}^{t_{i}}\left(t_{i}-s\right)^{\alpha-2}|f(s, u(s))| d s \\
& +\sum_{i=1}^{k-1} \frac{\left(t_{k}-t_{i}\right)}{\Gamma(\alpha-2)} \int_{t_{i-1}}^{t_{i}}\left(t_{i}-s\right)^{\alpha-3}|f(s, u(s))| d s \\
& +\sum_{i=1}^{k} \frac{\left(t-t_{k}\right)}{\Gamma(\alpha-2)} \int_{t_{i-1}}^{t_{i}}\left(t_{i}-s\right)^{\alpha-3}|f(s, u(s))| d s \\
& +\sum_{i=1}^{k}\left|I_{i}\left(u\left(t_{i}\right)\right)\right|+\sum_{i=1}^{k-1}\left(t_{k}-t_{i}\right)\left|I_{i}^{*}\left(u\left(t_{i}\right)\right)\right|+\sum_{i=1}^{k}\left(t-t_{k}\right)\left|I_{i}^{*}\left(u\left(t_{i}\right)\right)\right|+\frac{2 t}{1-\beta \eta^{2}} \\
& \times\left\{\frac{\beta}{\Gamma(\alpha)} \int_{t_{m}}^{\eta}(\eta-s)^{\alpha-1}|f(s, u(s))| d s+\sum_{i=1}^{m} \frac{\beta}{\Gamma(\alpha)} \int_{t_{i-1}}^{t_{i}}\left(t_{i}-s\right)^{\alpha-1}|f(s, u(s))| d s\right. \\
& +\sum_{i=1}^{m-1} \frac{\beta\left(t_{m}-t_{i}\right)}{\Gamma(\alpha-1)} \int_{t_{i-1}}^{t_{i}}\left(t_{i}-s\right)^{\alpha-2}|f(s, u(s))| d s \\
& +\sum_{i=1}^{m-1} \frac{\beta\left(t_{m}-t_{i}\right)^{2}}{2 \Gamma(\alpha-2)} \int_{t_{i-1}}^{t_{i}}\left(t_{i}-s\right)^{\alpha-3}|f(s, u(s))| d s \\
& +\sum_{i=1}^{m} \frac{\beta\left(\eta-t_{m}\right)}{\Gamma(\alpha-1)} \int_{t_{i-1}}^{t_{i}}\left(t_{i}-s\right)^{\alpha-2}|f(s, u(s))| d s \\
& +\sum_{i=1}^{m-1} \frac{\beta\left(\eta-t_{m}\right)\left(t_{m}-t_{i}\right)}{\Gamma(\alpha-2)} \int_{t_{i-1}}^{t_{i}}\left(t_{i}-s\right)^{\alpha-3}|f(s, u(s))| d s \\
& +\sum_{i=1}^{m} \frac{\beta\left(\eta-t_{m}\right)^{2}}{2 \Gamma(\alpha-2)} \int_{t_{i-1}}^{t_{i}}\left(t_{i}-s\right)^{\alpha-3}|f(s, u(s))| d s \\
& +\frac{1}{\Gamma(\alpha)} \sum_{i=1}^{p+1} \int_{t_{i-1}}^{t_{i}}\left(t_{i}-s\right)^{\alpha-1}|f(s, u(s))| d s \\
& +\sum_{i=1}^{p-1} \frac{\left(t_{p}-t_{i}\right)}{\Gamma(\alpha-1)} \int_{t_{i-1}}^{t_{i}}\left(t_{i}-s\right)^{\alpha-2}|f(s, u(s))| d s \\
& +\sum_{i=1}^{p-1} \frac{\left(t_{p}-t_{i}\right)^{2}}{2 \Gamma(\alpha-2)} \int_{t_{i-1}}^{t_{i}}\left(t_{i}-s\right)^{\alpha-3}|f(s, u(s))| d s \\
& +\sum_{i=1}^{p} \frac{\left(1-t_{p}\right)}{\Gamma(\alpha-1)} \int_{t_{i-1}}^{t_{i}}\left(t_{i}-s\right)^{\alpha-2}|f(s, u(s))| d s \\
& +\sum_{i=1}^{p-1} \frac{\left(1-t_{p}\right)\left(t_{p}-t_{i}\right)}{\Gamma(\alpha-2)} \int_{t_{i-1}}^{t_{i}}\left(t_{i}-s\right)^{\alpha-3}|f(s, u(s))| d s
\end{aligned}
$$




$$
\begin{aligned}
& +\sum_{i=1}^{p} \frac{\left(1-t_{p}\right)^{2}}{2 \Gamma(\alpha-2)} \int_{t_{i-1}}^{t_{i}}\left(t_{i}-s\right)^{\alpha-3}|f(s, u(s))| d s+\sum_{i=1}^{m-1} \beta\left(t_{m}-t_{i}\right)\left|I_{i}\left(u\left(t_{i}\right)\right)\right| \\
& +\sum_{i=1}^{m-1} \frac{\beta\left(t_{m}-t_{i}\right)^{2}}{2}\left|I_{i}^{*}\left(u\left(t_{i}\right)\right)\right|+\sum_{i=1}^{m} \beta\left(\eta-t_{m}\right)\left|I_{i}\left(u\left(t_{i}\right)\right)\right| \\
& +\sum_{i=1}^{m-1} \beta\left(\eta-t_{m}\right)\left(t_{m}-t_{i}\right)\left|I_{i}^{*}\left(u\left(t_{i}\right)\right)\right| \\
& +\sum_{i=1}^{m} \frac{\beta\left(\eta-t_{m}\right)^{2}}{2}\left|I_{i}^{*}\left(u\left(t_{i}\right)\right)\right|+\sum_{i=1}^{p-1}\left(t_{p}-t_{i}\right)\left|I_{i}\left(u\left(t_{i}\right)\right)\right|+\sum_{i=1}^{p-1} \frac{\left(t_{p}-t_{i}\right)^{2}}{2}\left|I_{i}^{*}\left(u\left(t_{i}\right)\right)\right| \\
& +\sum_{i=1}^{p}\left(1-t_{p}\right)\left|I_{i}\left(u\left(t_{i}\right)\right)\right|+\sum_{i=1}^{p-1}\left(1-t_{p}\right)\left(t_{p}-t_{i}\right)\left|I_{i}^{*}\left(u\left(t_{i}\right)\right)\right| \\
& \left.+\sum_{i=1}^{p} \frac{\left(1-t_{p}\right)^{2}}{2}\left|I_{i}^{*}\left(u\left(t_{i}\right)\right)\right|\right\} \\
& \leq \frac{L_{1}}{\Gamma(\alpha-1)} \int_{t_{k}}^{t}(t-s)^{\alpha-2} d s+\sum_{i=1}^{p} \frac{L_{1}}{\Gamma(\alpha-1)} \int_{t_{i-1}}^{t_{i}}\left(t_{i}-s\right)^{\alpha-2} d s \\
& +\sum_{i=1}^{p-1} \frac{L_{1}}{\Gamma(\alpha-2)} \int_{t_{i-1}}^{t_{i}}\left(t_{i}-s\right)^{\alpha-3} d s+\sum_{i=1}^{p} \frac{L_{1}}{\Gamma(\alpha-2)} \int_{t_{i-1}}^{t_{i}}\left(t_{i}-s\right)^{\alpha-3} d s+\sum_{i=1}^{p} L_{2}+\sum_{i=1}^{p-1} L_{3} \\
& +\sum_{i=1}^{p} L_{3}+\frac{2}{1-\beta \eta^{2}} \\
& \times\left\{\frac{L_{1} \beta}{\Gamma(\alpha)} \int_{t_{m}}^{\eta}(\eta-s)^{\alpha-1} d s+\sum_{i=1}^{p} \frac{L_{1} \beta}{\Gamma(\alpha)} \int_{t_{i-1}}^{t_{i}}\left(t_{i}-s\right)^{\alpha-1} d s\right. \\
& +\sum_{i=1}^{p-1} \frac{L_{1} \beta}{\Gamma(\alpha-1)} \int_{t_{i-1}}^{t_{i}}\left(t_{i}-s\right)^{\alpha-2} d s+\sum_{i=1}^{p-1} \frac{L_{1} \beta}{2 \Gamma(\alpha-2)} \int_{t_{i-1}}^{t_{i}}\left(t_{i}-s\right)^{\alpha-3} d s \\
& +\sum_{i=1}^{p} \frac{L_{1} \beta}{\Gamma(\alpha-1)} \int_{t_{i-1}}^{t_{i}}\left(t_{i}-s\right)^{\alpha-2} d s+\sum_{i=1}^{p-1} \frac{L_{1} \beta}{\Gamma(\alpha-2)} \int_{t_{i-1}}^{t_{i}}\left(t_{i}-s\right)^{\alpha-3} d s \\
& +\sum_{i=1}^{p} \frac{L_{1} \beta}{2 \Gamma(\alpha-2)} \int_{t_{i-1}}^{t_{i}}\left(t_{i}-s\right)^{\alpha-3} d s+\sum_{i=1}^{p+1} \frac{L_{1}}{\Gamma(\alpha)} \int_{t_{i-1}}^{t_{i}}\left(t_{i}-s\right)^{\alpha-1} d s \\
& +\sum_{i=1}^{p-1} \frac{L_{1}}{\Gamma(\alpha-1)} \int_{t_{i-1}}^{t_{i}}\left(t_{i}-s\right)^{\alpha-2} d s+\sum_{i=1}^{p-1} \frac{L_{1}}{2 \Gamma(\alpha-2)} \int_{t_{i-1}}^{t_{i}}\left(t_{i}-s\right)^{\alpha-3} d s \\
& +\sum_{i=1}^{p} \frac{L_{1}}{\Gamma(\alpha-1)} \int_{t_{i-1}}^{t_{i}}\left(t_{i}-s\right)^{\alpha-2} d s+\sum_{i=1}^{p-1} \frac{L_{1}}{\Gamma(\alpha-2)} \int_{t_{i-1}}^{t_{i}}\left(t_{i}-s\right)^{\alpha-3} d s \\
& +\sum_{i=1}^{p} \frac{L_{1}}{2 \Gamma(\alpha-2)} \int_{t_{i-1}}^{t_{i}}\left(t_{i}-s\right)^{\alpha-3} d s+\sum_{i=1}^{p-1} \beta L_{2}+\sum_{i=1}^{p-1} \frac{\beta}{2} L_{3}+\sum_{i=1}^{p} \beta L_{2}+\sum_{i=1}^{p-1} \beta L_{3} \\
& \left.+\sum_{i=1}^{p} \frac{\beta}{2} L_{3}+\sum_{i=1}^{p-1} L_{2}+\sum_{i=1}^{p-1} \frac{1}{2} L_{3}+\sum_{i=1}^{p} L_{2}+\sum_{i=1}^{p-1} L_{3}+\sum_{i=1}^{p} \frac{1}{2} L_{3}\right\}
\end{aligned}
$$




$$
\begin{aligned}
& \leq \frac{L_{1}}{\Gamma(\alpha)}+\frac{p L_{1}}{\Gamma(\alpha)}+\frac{(p-1) L_{1}}{\Gamma(\alpha-1)}+\frac{p L_{1}}{\Gamma(\alpha-1)} p L_{2}+(p-1) L_{3}+p L_{3}+\frac{2}{1-\beta \eta^{2}} \\
& \quad \times\left\{\frac{\beta L_{1}}{\Gamma(\alpha+1)}+\frac{\beta p L_{1}}{\Gamma(\alpha+1)}+\frac{\beta(p-1) L_{1}}{\Gamma(\alpha)}+\frac{\beta(p-1) L_{1}}{2 \Gamma(\alpha-1)}+\frac{\beta p L_{1}}{\Gamma(\alpha)}+\frac{\beta(p-1) L_{1}}{\Gamma(\alpha-1)}\right. \\
& \quad+\frac{\beta p L_{1}}{2 \Gamma(\alpha-1)}+\frac{(p+1) L_{1}}{\Gamma(\alpha+1)}+\frac{(p-1) L_{1}}{\Gamma(\alpha)}+\frac{(p-1) L_{1}}{2 \Gamma(\alpha-1)}+\frac{p L_{1}}{\Gamma(\alpha)}+\frac{(p-1) L_{1}}{\Gamma(\alpha-1)} \\
& \quad+\frac{p L_{1}}{2 \Gamma(\alpha-1)}+\beta(p-1) L_{2}+\frac{\beta(p-1)}{2} L_{3}+\beta p L_{2}+\beta(p-1) L_{3}+\frac{\beta p}{2} L_{3} \\
& \left.\quad+(p-1) L_{2}+\frac{p-1}{2} L_{3}+p L_{2}+(p-1) L_{3}+\frac{p}{2} L_{3}\right\} \\
& =\frac{(p+1) L_{1}+\frac{(2 p-1) L_{1}}{\Gamma(\alpha)} p L_{2}+(2 p-1) L_{3}+\frac{2(\beta+1)}{1-\beta \eta^{2}}}{\Gamma(\alpha-1)} \\
& \quad \times\left\{\frac{(p+1) L_{1}}{\Gamma(\alpha+1)}+\frac{(2 p-1) L_{1}}{\Gamma(\alpha)}+\frac{(4 p-3) L_{1}}{2 \Gamma(\alpha-1)}+(2 p-1) L_{2}+\left(2 p-\frac{3}{2}\right) L_{3}\right\}:=\bar{L} .
\end{aligned}
$$

Hence, let $t_{1}, t_{2} \in J_{k}, t_{1}<t_{2}, 0 \leq k \leq p$, we have

$$
\left|(A u)\left(t_{2}\right)-(A u)\left(t_{1}\right)\right| \leq \int_{t_{1}}^{t_{2}}\left|(A u)^{\prime}(s)\right| d s \leq \bar{L}\left(t_{2}-t_{1}\right)
$$

This implies that $A$ is equicontinuous on all $J_{k}, k=0,1,2, \ldots, p$. The Arzela-Ascoli Theorem implies that $A: C(J) \rightarrow C(J)$ is completely continuous.

Theorem 3.2. Assume that the nonlinearity $f$ is bounded and that the impulse functions $I_{k}, I_{k^{\prime}}^{*} k=$ $1,2, \ldots, p$ are bounded. Then, the nonlinear impulsive fractional three-point boundary value problems (1.1) have at least one solution.

Proof. Firstly, by Lemma 3.1, we know that the operator $A: C(J) \rightarrow C(J)$ is completely continuous.

Let $L_{i}(i=1,2,3)$ be nonnegative constants such that

$$
|f(t, u)| \leq L_{1}, \quad\left|I_{k}(u)\right| \leq L_{2}, \quad\left|I_{k}^{*}(u)\right| \leq L_{3}, \quad t \in J, u \in R .
$$

For $0<\mu<1$, consider the equation:

$$
u=\mu A u .
$$


If $u$ is a solution of (3.7), then for every $t \in J$ we have that

$$
\begin{aligned}
& |u(t)|=\mu|A u(t)| \\
& \leq \frac{1}{\Gamma(\alpha)} \int_{t_{k}}^{t}(t-s)^{\alpha-1}|f(s, u(s))| d s+\frac{1}{\Gamma(\alpha)} \sum_{i=1}^{k} \int_{t_{i-1}}^{t_{i}}\left(t_{i}-s\right)^{\alpha-1}|f(s, u(s))| d s \\
& +\sum_{i=1}^{k-1} \frac{\left(t_{k}-t_{i}\right)}{\Gamma(\alpha-1)} \int_{t_{i-1}}^{t_{i}}\left(t_{i}-s\right)^{\alpha-2}|f(s, u(s))| d s \\
& +\sum_{i=1}^{k-1} \frac{\left(t_{k}-t_{i}\right)^{2}}{2 \Gamma(\alpha-2)} \int_{t_{i-1}}^{t_{i}}\left(t_{i}-s\right)^{\alpha-3}|f(s, u(s))| d s \\
& +\sum_{i=1}^{k} \frac{\left(t-t_{k}\right)}{\Gamma(\alpha-1)} \int_{t_{i-1}}^{t_{i}}\left(t_{i}-s\right)^{\alpha-2}|f(s, u(s))| d s \\
& +\sum_{i=1}^{k-1} \frac{\left(t-t_{k}\right)\left(t_{k}-t_{i}\right)}{\Gamma(\alpha-2)} \int_{t_{i-1}}^{t_{i}}\left(t_{i}-s\right)^{\alpha-3}|f(s, u(s))| d s \\
& +\sum_{i=1}^{k} \frac{\left(t-t_{k}\right)^{2}}{2 \Gamma(\alpha-2)} \int_{t_{i-1}}^{t_{i}}\left(t_{i}-s\right)^{\alpha-3}|f(s, u(s))| d s \\
& +\sum_{i=1}^{k-1}\left(t_{k}-t_{i}\right)\left|I_{i}\left(u\left(t_{i}\right)\right)\right|+\sum_{i=1}^{k-1} \frac{\left(t_{k}-t_{i}\right)^{2}}{2}\left|I_{i}^{*}\left(u\left(t_{i}\right)\right)\right| \\
& +\sum_{i=1}^{k}\left(t-t_{k}\right)\left|I_{i}\left(u\left(t_{i}\right)\right)\right|+\sum_{i=1}^{k-1}\left(t-t_{k}\right)\left(t_{k}-t_{i}\right)\left|I_{i}^{*}\left(u\left(t_{i}\right)\right)\right| \\
& +\sum_{i=1}^{k} \frac{\left(t-t_{k}\right)^{2}}{2}\left|I_{i}^{*}\left(u\left(t_{i}\right)\right)\right|+\frac{t^{2}}{1-\beta \eta^{2}} \\
& \times\left\{\frac{\beta}{\Gamma(\alpha)} \int_{t_{m}}^{\eta}(\eta-s)^{\alpha-1}|f(s, u(s))| d s+\sum_{i=1}^{m} \frac{\beta}{\Gamma(\alpha)} \int_{t_{i-1}}^{t_{i}}\left(t_{i}-s\right)^{\alpha-1}|f(s, u(s))| d s\right. \\
& +\sum_{i=1}^{m-1} \frac{\beta\left(t_{m}-t_{i}\right)}{\Gamma(\alpha-1)} \int_{t_{i-1}}^{t_{i}}\left(t_{i}-s\right)^{\alpha-2}|f(s, u(s))| d s \\
& +\sum_{i=1}^{m-1} \frac{\beta\left(t_{m}-t_{i}\right)^{2}}{2 \Gamma(\alpha-2)} \int_{t_{i-1}}^{t_{i}}\left(t_{i}-s\right)^{\alpha-3}|f(s, u(s))| d s \\
& +\sum_{i=1}^{m} \frac{\beta\left(\eta-t_{m}\right)}{\Gamma(\alpha-1)} \int_{t_{i-1}}^{t_{i}}\left(t_{i}-s\right)^{\alpha-2}|f(s, u(s))| d s \\
& +\sum_{i=1}^{m-1} \frac{\beta\left(\eta-t_{m}\right)\left(t_{m}-t_{i}\right)}{\Gamma(\alpha-2)} \int_{t_{i-1}}^{t_{i}}\left(t_{i}-s\right)^{\alpha-3}|f(s, u(s))| d s \\
& +\sum_{i=1}^{m} \frac{\beta\left(\eta-t_{m}\right)^{2}}{2 \Gamma(\alpha-2)} \int_{t_{i-1}}^{t_{i}}\left(t_{i}-s\right)^{\alpha-3}|f(s, u(s))| d s
\end{aligned}
$$




$$
\begin{aligned}
+ & \frac{1}{\Gamma(\alpha)} \sum_{i=1}^{p+1} \int_{t_{i-1}}^{t_{i}}\left(t_{i}-s\right)^{\alpha-1}|f(s, u(s))| d s \\
& +\sum_{i=1}^{p-1} \frac{\left(t_{p}-t_{i}\right)}{\Gamma(\alpha-1)} \int_{t_{i-1}}^{t_{i}}\left(t_{i}-s\right)^{\alpha-2}|f(s, u(s))| d s \\
& +\sum_{i=1}^{p-1} \frac{\left(t_{p}-t_{i}\right)^{2}}{2 \Gamma(\alpha-2)} \int_{t_{i-1}}^{t_{i}}\left(t_{i}-s\right)^{\alpha-3}|f(s, u(s))| d s \\
& +\sum_{i=1}^{p} \frac{\left(1-t_{p}\right)}{\Gamma(\alpha-1)} \int_{t_{i-1}}^{t_{i}}\left(t_{i}-s\right)^{\alpha-2}|f(s, u(s))| d s \\
& +\sum_{i=1}^{p-1} \frac{\left(1-t_{p}\right)\left(t_{p}-t_{i}\right)}{\Gamma(\alpha-2)} \int_{t_{i-1}}^{t_{i}}\left(t_{i}-s\right)^{\alpha-3}|f(s, u(s))| d s \\
+ & \sum_{i=1}^{p} \frac{\left(1-t_{p}\right)^{2}}{2 \Gamma(\alpha-2)} \int_{t_{i-1}}^{t_{i}}\left(t_{i}-s\right)^{\alpha-3}|f(s, u(s))| d s+\sum_{i=1}^{m-1} \beta\left(t_{m}-t_{i}\right)\left|I_{i}\left(u\left(t_{i}\right)\right)\right| \\
& +\sum_{i=1}^{m-1} \frac{\beta\left(t_{m}-t_{i}\right)^{2}}{2}\left|I_{i}^{*}\left(u\left(t_{i}\right)\right)\right|+\sum_{i=1}^{m} \beta\left(\eta-t_{m}\right)\left|I_{i}\left(u\left(t_{i}\right)\right)\right| \\
+ & \beta\left(1-\eta^{2}\right) \\
+ & -\beta \eta^{2}\left\{\frac{(p+1)}{\Gamma(\alpha+1)}+\frac{(2 p-1) L_{1}}{\Gamma-1}+\frac{(4 p-3) L_{1}}{2 \Gamma(\alpha-1)}+(2 p-1) L_{2}+\left(2 p-\frac{3}{2}\right) L_{3}\right\}, \\
+ & \sum_{i=1}^{m} \beta\left(\eta-t_{m}\right)\left(t_{m}-t_{i}\right)\left|I_{i}^{*}\left(u\left(t_{i}\right)\right)\right| \\
+ & \sum_{i=1}^{p}\left(1-t_{p}\right)\left|I_{i}\left(u\left(t_{i}\right)\right)\right|+\sum_{i=1}^{p-1}\left(1-t_{p}\right)\left(t_{p}-t_{i}\right)\left|I_{i}^{*}\left(u\left(t_{i}\right)\right)\right| \\
+ & \sum_{i=1}^{m} \frac{\beta\left(\eta-t_{m}\right)^{2}}{2}\left|I_{i}^{*}\left(u\left(t_{i}\right)\right)\right|+\sum_{i=1}^{p-1}\left(t_{p}-t_{i}\right)\left|I_{i}\left(u\left(t_{i}\right)\right)\right| \\
& +\sum_{i=1}^{p-1} \frac{\left(t_{p}-t_{i}\right)^{2}}{2}\left|I_{i}^{*}\left(u\left(t_{i}\right)\right)\right|
\end{aligned}
$$

which implies for any $t \in J$, it holds that

$$
\|u\| \leq \frac{2+\beta\left(1-\eta^{2}\right)}{1-\beta \eta^{2}}\left\{\frac{(p+1) L_{1}}{\Gamma(\alpha+1)}+\frac{(2 p-1) L_{1}}{\Gamma(\alpha)}+\frac{(4 p-3) L_{1}}{2 \Gamma(\alpha-1)}+(2 p-1) L_{2}+\left(2 p-\frac{3}{2}\right) L_{3}\right\} .
$$


This shows that all the solutions of (3.7) are bounded independently of $0<\mu<1$. Using Schaeffer's theorem (see Theorem 2.2), we get that $A$ has at least one fixed point, which implies that (1.1) has at least one solution.

Now, we present some existence results when the nonlinearity and the impulse functions have sublinear growth.

Theorem 3.3. Assume that

$\left(\mathrm{H}_{1}\right)$ there exist nonnegative constants $a$ and $b$ such that

$$
|f(t, u)| \leq a+b|u|^{\rho}, \quad 0 \leq \rho<1, t \in J, u \in \mathbb{R}
$$

$\left(\mathrm{H}_{2}\right)$ there exist nonnegative constants $a_{k}, b_{k}, a_{k}^{*}, b_{k}^{*}$ such that

$$
\left|I_{k}(u)\right| \leq a_{k}+b_{k}|u|^{\rho_{k}}, \quad\left|I_{k}^{*}(u)\right| \leq a_{k}^{*}+b_{k}^{*}|u|^{\rho_{k}^{*}}, \quad 0 \leq \rho_{k}, \rho_{k}^{*}<1, u \in \mathbb{R}, k=1,2, \ldots, p .
$$

Then, problem (1.1) has at least one solution.

Proof. If $u \in C(J)$, then we can write that

$$
\begin{gathered}
|f(s, u(s))| \leq a+b|u(s)|^{\rho}, \quad s \in J, \\
\left|I_{k}\left(u\left(t_{k}\right)\right)\right| \leq a_{k}+b_{k}\left|u\left(t_{k}\right)\right|^{\rho_{k}}, \quad\left|I_{k}^{*}\left(u\left(t_{k}\right)\right)\right| \leq a_{k}^{*}+b_{k}^{*}\left|u\left(t_{k}\right)\right|^{\rho_{k}^{*}}, \quad k=1,2, \ldots, p .
\end{gathered}
$$

So, if $u$ is a solution of (3.7), then, by the similar process used to obtain (3.2), for every $t \in J$ we have that

$$
\begin{aligned}
|u(t)| \leq & \frac{\mu}{\Gamma(\alpha)} \int_{t_{k}}^{t}(t-s)^{\alpha-1}|f(s, u(s))| d s+\frac{\mu}{\Gamma(\alpha)} \sum_{i=1}^{k} \int_{t_{i-1}}^{t_{i}}\left(t_{i}-s\right)^{\alpha-1}|f(s, u(s))| d s \\
& +\sum_{i=1}^{k-1} \frac{\mu\left(t_{k}-t_{i}\right)}{\Gamma(\alpha-1)} \int_{t_{i-1}}^{t_{i}}\left(t_{i}-s\right)^{\alpha-2}|f(s, u(s))| d s \\
& +\sum_{i=1}^{k-1} \frac{\mu\left(t_{k}-t_{i}\right)^{2}}{2 \Gamma(\alpha-2)} \int_{t_{i-1}}^{t_{i}}\left(t_{i}-s\right)^{\alpha-3}|f(s, u(s))| d s+\sum_{i=1}^{k} \frac{\mu\left(t-t_{k}\right)}{\Gamma(\alpha-1)} \int_{t_{i-1}}^{t_{i}}\left(t_{i}-s\right)^{\alpha-2}|f(s, u(s))| d s \\
& +\sum_{i=1}^{k-1} \frac{\mu\left(t-t_{k}\right)\left(t_{k}-t_{i}\right)}{\Gamma(\alpha-2)} \int_{t_{i-1}}^{t_{i}}\left(t_{i}-s\right)^{\alpha-3}|f(s, u(s))| d s \\
& +\sum_{i=1}^{k} \frac{\mu\left(t-t_{k}\right)^{2}}{2 \Gamma(\alpha-2)} \int_{t_{i-1}}^{t_{i}}\left(t_{i}-s\right)^{\alpha-3}|f(s, u(s))| d s+\sum_{i=1}^{k-1} \mu\left(t_{k}-t_{i}\right)\left|I_{i}\left(u\left(t_{i}\right)\right)\right| \\
& +\sum_{i=1}^{k-1} \frac{\mu\left(t_{k}-t_{i}\right)^{2}}{2}\left|I_{i}^{*}\left(u\left(t_{i}\right)\right)\right|+\sum_{i=1}^{k} \mu\left(t-t_{k}\right)\left|I_{i}\left(u\left(t_{i}\right)\right)\right|+\sum_{i=1}^{k-1} \mu\left(t-t_{k}\right)\left(t_{k}-t_{i}\right)\left|I_{i}^{*}\left(u\left(t_{i}\right)\right)\right|
\end{aligned}
$$




$$
\begin{aligned}
& +\sum_{i=1}^{k} \frac{\mu\left(t-t_{k}\right)^{2}}{2}\left|I_{i}^{*}\left(u\left(t_{i}\right)\right)\right|+\frac{\mu t^{2}}{1-\beta \eta^{2}} \\
& \times\left\{\frac{\beta}{\Gamma(\alpha)} \int_{t_{m}}^{\eta}(\eta-s)^{\alpha-1}|f(s, u(s))| d s\right. \\
& +\sum_{i=1}^{m} \frac{\beta}{\Gamma(\alpha)} \int_{t_{i-1}}^{t_{i}}\left(t_{i}-s\right)^{\alpha-1}|f(s, u(s))| d s+\sum_{i=1}^{m-1} \frac{\beta\left(t_{m}-t_{i}\right)}{\Gamma(\alpha-1)} \int_{t_{i-1}}^{t_{i}}\left(t_{i}-s\right)^{\alpha-2}|f(s, u(s))| d s \\
& +\sum_{i=1}^{m-1} \frac{\beta\left(t_{m}-t_{i}\right)^{2}}{2 \Gamma(\alpha-2)} \int_{t_{i-1}}^{t_{i}}\left(t_{i}-s\right)^{\alpha-3}|f(s, u(s))| d s \\
& +\sum_{i=1}^{m} \frac{\beta\left(\eta-t_{m}\right)}{\Gamma(\alpha-1)} \int_{t_{i-1}}^{t_{i}}\left(t_{i}-s\right)^{\alpha-2}|f(s, u(s))| d s \\
& +\sum_{i=1}^{m-1} \frac{\beta\left(\eta-t_{m}\right)\left(t_{m}-t_{i}\right)}{\Gamma(\alpha-2)} \int_{t_{i-1}}^{t_{i}}\left(t_{i}-s\right)^{\alpha-3}|f(s, u(s))| d s \\
& +\sum_{i=1}^{m} \frac{\beta\left(\eta-t_{m}\right)^{2}}{2 \Gamma(\alpha-2)} \int_{t_{i-1}}^{t_{i}}\left(t_{i}-s\right)^{\alpha-3}|f(s, u(s))| d s+\frac{1}{\Gamma(\alpha)} \sum_{i=1}^{p+1} \int_{t_{i-1}}^{t_{i}}\left(t_{i}-s\right)^{\alpha-1}|f(s, u(s))| d s \\
& +\sum_{i=1}^{p-1} \frac{\left(t_{p}-t_{i}\right)}{\Gamma(\alpha-1)} \int_{t_{i-1}}^{t_{i}}\left(t_{i}-s\right)^{\alpha-2}|f(s, u(s))| d s \\
& +\sum_{i=1}^{p-1} \frac{\left(t_{p}-t_{i}\right)^{2}}{2 \Gamma(\alpha-2)} \int_{t_{i-1}}^{t_{i}}\left(t_{i}-s\right)^{\alpha-3}|f(s, u(s))| d s \\
& +\sum_{i=1}^{p} \frac{\left(1-t_{p}\right)}{\Gamma(\alpha-1)} \int_{t_{i-1}}^{t_{i}}\left(t_{i}-s\right)^{\alpha-2}|f(s, u(s))| d s \\
& +\sum_{i=1}^{p-1} \frac{\left(1-t_{p}\right)\left(t_{p}-t_{i}\right)}{\Gamma(\alpha-2)} \int_{t_{i-1}}^{t_{i}}\left(t_{i}-s\right)^{\alpha-3}|f(s, u(s))| d s \\
& +\sum_{i=1}^{p} \frac{\left(1-t_{p}\right)^{2}}{2 \Gamma(\alpha-2)} \int_{t_{i-1}}^{t_{i}}\left(t_{i}-s\right)^{\alpha-3}|f(s, u(s))| d s+\sum_{i=1}^{m-1} \beta\left(t_{m}-t_{i}\right)\left|I_{i}\left(u\left(t_{i}\right)\right)\right| \\
& +\sum_{i=1}^{m-1} \frac{\beta\left(t_{m}-t_{i}\right)^{2}}{2}\left|I_{i}^{*}\left(u\left(t_{i}\right)\right)\right|+\sum_{i=1}^{m} \beta\left(\eta-t_{m}\right)\left|I_{i}\left(u\left(t_{i}\right)\right)\right| \\
& +\sum_{i=1}^{m-1} \beta\left(\eta-t_{m}\right)\left(t_{m}-t_{i}\right)\left|I_{i}^{*}\left(u\left(t_{i}\right)\right)\right| \\
& +\sum_{i=1}^{m} \frac{\beta\left(\eta-t_{m}\right)^{2}}{2}\left|I_{i}^{*}\left(u\left(t_{i}\right)\right)\right|+\sum_{i=1}^{p-1}\left(t_{p}-t_{i}\right)\left|I_{i}\left(u\left(t_{i}\right)\right)\right|+\sum_{i=1}^{p-1} \frac{\left(t_{p}-t_{i}\right)^{2}}{2}\left|I_{i}^{*}\left(u\left(t_{i}\right)\right)\right| \\
& \left.+\sum_{i=1}^{p}\left(1-t_{p}\right)\left|I_{i}\left(u\left(t_{i}\right)\right)\right|+\sum_{i=1}^{p-1}\left(1-t_{p}\right)\left(t_{p}-t_{i}\right)\left|I_{i}^{*}\left(u\left(t_{i}\right)\right)\right|+\sum_{i=1}^{p} \frac{\left(1-t_{p}\right)^{2}}{2}\left|I_{i}^{*}\left(u\left(t_{i}\right)\right)\right|\right\}
\end{aligned}
$$




$$
\begin{aligned}
\leq & M_{1} a+M_{2}\left(\sum_{k=1}^{p} a_{k}+\sum_{k=1}^{p-1} a_{k}\right)+\frac{M_{2}}{2}\left(\sum_{k=1}^{p} a_{k}^{*}+3 \sum_{k=1}^{p-1} a_{k}^{*}\right)+M_{1} b\|u\|^{\rho}+M_{2} \sum_{k=1}^{p} b_{k}\|u\|^{\rho_{k}} \\
& +M_{2} \sum_{k=1}^{p-1} b_{k}\|u\|^{\rho_{k}}+\frac{M_{2}}{2} \sum_{k=1}^{p} b_{k}^{*}\|u\|^{\rho_{k}^{*}}+\frac{3 M_{2}}{2} \sum_{k=1}^{p-1} b_{k}^{*}\|u\|^{\rho_{k}^{*}},
\end{aligned}
$$

where

$$
M_{1}=\frac{2+\beta\left(1-\eta^{2}\right)}{1-\beta \eta^{2}}\left(\frac{(p+1)}{\Gamma(\alpha+1)}+\frac{(2 p-1)}{\Gamma(\alpha)}+\frac{(4 p-3)}{2 \Gamma(\alpha-1)}\right), \quad M_{2}=\frac{2+\beta\left(1-\eta^{2}\right)}{1-\beta \eta^{2}} .
$$

In consequence,

$$
\|u\| \leq M+M_{1} b\|u\|^{\rho}+M_{2} \sum_{k=1}^{p} b_{k}\|u\|^{\rho_{k}}+M_{2} \sum_{k=1}^{p-1} b_{k}\|u\|^{\rho_{k}}+\frac{M_{2}}{2} \sum_{k=1}^{p} b_{k}^{*}\|u\|^{\rho_{k}^{*}}+\frac{3 M_{2}}{2} \sum_{k=1}^{p-1} b_{k}^{*}\|u\|^{*}
$$

where $M=M_{1} a+M_{2}\left(\sum_{k=1}^{p} a_{k}+\sum_{k=1}^{p-1} a_{k}\right)+\left(M_{2} / 2\right)\left(\sum_{k=1}^{p} a_{k}^{*}+3 \sum_{k=1}^{p-1} a_{k}^{*}\right)$.

Now, taking into account that $0 \leq \rho, \rho_{k}, \rho_{k}^{*}<1, k=1,2, \ldots, p$, we can conclude that there exists a constant $C>0$ such that $\|u\| \leq C$ for any solution of (3.7), $0<\mu<1$. Therefore, by Schaeffer's theorem (Theorem 2.2), we obtain the existence of a fixed point for $A$, which implies the problem (1.1) has at least one solution.

Theorem 3.4. Assume that

$\left(\mathrm{H}_{3}\right)$ there exist nonnegative constants $a$ and $b$ such that

$$
|f(t, u)| \leq a+b|u|, \quad t \in J, u \in \mathbb{R}
$$

$\left(\mathrm{H}_{4}\right)$ there exist nonnegative constants $a_{k}, b_{k}, a_{k}^{*}, b_{k}^{*}$ such that

$$
\left|I_{k}(u)\right| \leq a_{k}+b_{k}|u|, \quad\left|I_{k}^{*}(u)\right| \leq a_{k}^{*}+b_{k}^{*}|u|, \quad u \in \mathbb{R}, k=1,2, \ldots, p .
$$

If

$$
M_{1} b+M_{2}\left(\sum_{k=1}^{p}\left(b_{k}+\frac{1}{2} b_{k}^{*}\right)+\sum_{k=1}^{p-1}\left(b_{k}+\frac{3}{2} b_{k}^{*}\right)\right)<1
$$

where

$$
M_{1}=\frac{2+\beta\left(1-\eta^{2}\right)}{1-\beta \eta^{2}}\left(\frac{(p+1)}{\Gamma(\alpha+1)}+\frac{(2 p-1)}{\Gamma(\alpha)}+\frac{(4 p-3)}{2 \Gamma(\alpha-1)}\right), \quad M_{2}=\frac{2+\beta\left(1-\eta^{2}\right)}{1-\beta \eta^{2}} .
$$

Then problem (1.1) has at least one solution. 
Proof. The proof is similar to that of Theorem 3.3, so we omit it.

Theorem 3.5. Let $\lim _{u \rightarrow 0} f(t, u) / u=0, \lim _{u \rightarrow 0} I_{k}(u) / u=0$, and $\lim _{u \rightarrow 0} I_{k}^{*}(u) / u=0$, then problem (1.1) has at least one solution.

Proof. Now, in view of $\lim _{u \rightarrow 0} f(t, u) / u=0, \lim _{u \rightarrow 0} I_{k}(u) / u=0$, and $\lim _{u \rightarrow 0} I_{k}^{*}(u) / u=0$, there exists a constant $r>0$ such that $|f(t, u)| \leq \delta_{1}|u|,\left|I_{k}(u)\right| \leq \delta_{2}|u|$, and $\left|I_{k}^{*}(u)\right| \leq \delta_{3}|u|$ for $0<|u|<r$, where $\delta_{i}>0(i=1,2,3)$ satisfy

$$
\frac{2+\beta\left(1-\eta^{2}\right)}{1-\beta \eta^{2}}\left\{\frac{(p+1) \delta_{1}}{\Gamma(\alpha+1)}+\frac{(2 p-1) \delta_{1}}{\Gamma(\alpha)}+\frac{(4 p-3) \delta_{1}}{2 \Gamma(\alpha-1)}+(2 p-1) \delta_{2}+\left(2 p-\frac{3}{2}\right) \delta_{3}\right\} \leq 1
$$

Let $\Omega=\{u \in C(J) \mid\|u\|<r\}$. Take $u \in C(J)$ such that $\|u\|=r$, that is, $u \in \partial \Omega$. By Lemma 3.1, we know that $A$ is completely continuous, and

$$
\begin{aligned}
|(A u)(t)| \leq & \frac{2+\beta\left(1-\eta^{2}\right)}{1-\beta \eta^{2}} \\
& \times\left\{\frac{(p+1) \delta_{1}}{\Gamma(\alpha+1)}+\frac{(2 p-1) \delta_{1}}{\Gamma(\alpha)}+\frac{(4 p-3) \delta_{1}}{2 \Gamma(\alpha-1)}+(2 p-1) \delta_{2}+\left(2 p-\frac{3}{2}\right) \delta_{3}\right\}\|u\| .
\end{aligned}
$$

Thus, in view of (3.21), we obtain $\|A u\| \leq\|u\|, u \in \partial \Omega$. Therefore, by Theorem 2.3, the operator $A$ has at least one fixed point, which implies that (1.1) has at least one solution $u \in \bar{\Omega}$.

For the forthcoming analysis, we need the following assumption:

$\left(\mathrm{H}_{5}\right)$ there exist positive constants $K_{i}(i=1,2,3)$ such that

$$
|f(t, u)-f(t, v)| \leq K_{1}|u-v|, \quad\left|I_{k}(u)-I_{k}(v)\right| \leq K_{2}|u-v|, \quad\left|I_{k}^{*}(u)-I_{k}^{*}(v)\right| \leq K_{3}|u-v|,
$$

for $t \in J, u, v \in R$ and $k=1,2, \ldots, p$.

Define the constants:

$$
\Lambda=\frac{2+\beta\left(1-\eta^{2}\right)}{1-\beta \eta^{2}}\left\{\frac{(p+1) K_{1}}{\Gamma(\alpha+1)}+\frac{(2 p-1) K_{1}}{\Gamma(\alpha)}+\frac{(4 p-3) K_{1}}{2 \Gamma(\alpha-1)}+(2 p-1) K_{2}+\left(2 p-\frac{3}{2}\right) K_{3}\right\}
$$

Theorem 3.6. If condition $\left(H_{5}\right)$ holds. Then problem (1.1) has a unique solution provided $\Lambda<1$, where $\Lambda$ is given by (3.23). 
Proof. Let $u, v \in C(J)$, by a simple computation, we can get

$$
\begin{aligned}
& |(A u)(t)-(A v)(t)| \\
& \leq \frac{1}{\Gamma(\alpha)} \int_{t_{k}}^{t}(t-s)^{\alpha-1}|f(s, u(s))-f(s, v(s))| d s \\
& +\frac{1}{\Gamma(\alpha)} \sum_{i=1}^{k} \int_{t_{i-1}}^{t_{i}}\left(t_{i}-s\right)^{\alpha-1}|f(s, u(s))-f(s, v(s))| d s \\
& +\sum_{i=1}^{k-1} \frac{\left(t_{k}-t_{i}\right)}{\Gamma(\alpha-1)} \int_{t_{i-1}}^{t_{i}}\left(t_{i}-s\right)^{\alpha-2}|f(s, u(s))-f(s, v(s))| d s \\
& +\sum_{i=1}^{k-1} \frac{\left(t_{k}-t_{i}\right)^{2}}{2 \Gamma(\alpha-2)} \int_{t_{i-1}}^{t_{i}}\left(t_{i}-s\right)^{\alpha-3}|f(s, u(s))-f(s, v(s))| d s \\
& +\sum_{i=1}^{k} \frac{\left(t-t_{k}\right)}{\Gamma(\alpha-1)} \int_{t_{i-1}}^{t_{i}}\left(t_{i}-s\right)^{\alpha-2}|f(s, u(s))-f(s, v(s))| d s \\
& +\sum_{i=1}^{k-1} \frac{\left(t-t_{k}\right)\left(t_{k}-t_{i}\right)}{\Gamma(\alpha-2)} \int_{t_{i-1}}^{t_{i}}\left(t_{i}-s\right)^{\alpha-3}|f(s, u(s))-f(s, v(s))| d s \\
& +\sum_{i=1}^{k} \frac{\left(t-t_{k}\right)^{2}}{2 \Gamma(\alpha-2)} \int_{t_{i-1}}^{t_{i}}\left(t_{i}-s\right)^{\alpha-3}|f(s, u(s))-f(s, v(s))| d s \\
& +\sum_{i=1}^{k-1}\left(t_{k}-t_{i}\right)\left|I_{i}\left(u\left(t_{i}\right)\right)-I_{i}\left(v\left(t_{i}\right)\right)\right|+\sum_{i=1}^{k-1} \frac{\left(t_{k}-t_{i}\right)^{2}}{2}\left|I_{i}^{*}\left(u\left(t_{i}\right)\right)-I_{i}^{*}\left(v\left(t_{i}\right)\right)\right| \\
& +\sum_{i=1}^{k}\left(t-t_{k}\right)\left|I_{i}\left(u\left(t_{i}\right)\right)-I_{i}\left(v\left(t_{i}\right)\right)\right|+\sum_{i=1}^{k-1}\left(t-t_{k}\right)\left(t_{k}-t_{i}\right)\left|I_{i}^{*}\left(u\left(t_{i}\right)\right)-I_{i}^{*}\left(v\left(t_{i}\right)\right)\right| \\
& +\sum_{i=1}^{k} \frac{\left(t-t_{k}\right)^{2}}{2}\left|I_{i}^{*}\left(u\left(t_{i}\right)\right)-I_{i}^{*}\left(v\left(t_{i}\right)\right)\right|+\frac{t^{2}}{1-\beta \eta^{2}} \\
& \times\left\{\frac{\beta}{\Gamma(\alpha)} \int_{t_{m}}^{\eta}(\eta-s)^{\alpha-1}|f(s, u(s))-f(s, v(s))| d s\right. \\
& +\sum_{i=1}^{m} \frac{\beta}{\Gamma(\alpha)} \int_{t_{i-1}}^{t_{i}}\left(t_{i}-s\right)^{\alpha-1}|f(s, u(s))-f(s, v(s))| d s \\
& +\sum_{i=1}^{m-1} \frac{\beta\left(t_{m}-t_{i}\right)}{\Gamma(\alpha-1)} \int_{t_{i-1}}^{t_{i}}\left(t_{i}-s\right)^{\alpha-2}|f(s, u(s))-f(s, v(s))| d s \\
& +\sum_{i=1}^{m-1} \frac{\beta\left(t_{m}-t_{i}\right)^{2}}{2 \Gamma(\alpha-2)} \int_{t_{i-1}}^{t_{i}}\left(t_{i}-s\right)^{\alpha-3}|f(s, u(s))-f(s, v(s))| d s \\
& +\sum_{i=1}^{m} \frac{\beta\left(\eta-t_{m}\right)}{\Gamma(\alpha-1)} \int_{t_{i-1}}^{t_{i}}\left(t_{i}-s\right)^{\alpha-2}|f(s, u(s))-f(s, v(s))| d s
\end{aligned}
$$




$$
\begin{aligned}
& +\sum_{i=1}^{m-1} \frac{\beta\left(\eta-t_{m}\right)\left(t_{m}-t_{i}\right)}{\Gamma(\alpha-2)} \int_{t_{i-1}}^{t_{i}}\left(t_{i}-s\right)^{\alpha-3}|f(s, u(s))-f(s, v(s))| d s \\
& +\sum_{i=1}^{m} \frac{\beta\left(\eta-t_{m}\right)^{2}}{2 \Gamma(\alpha-2)} \int_{t_{i-1}}^{t_{i}}\left(t_{i}-s\right)^{\alpha-3}|f(s, u(s))-f(s, v(s))| d s \\
& +\frac{1}{\Gamma(\alpha)} \sum_{i=1}^{p+1} \int_{t_{i-1}}^{t_{i}}\left(t_{i}-s\right)^{\alpha-1}|f(s, u(s))-f(s, v(s))| d s \\
& +\sum_{i=1}^{p-1} \frac{\left(t_{p}-t_{i}\right)}{\Gamma(\alpha-1)} \int_{t_{i-1}}^{t_{i}}\left(t_{i}-s\right)^{\alpha-2}|f(s, u(s))-f(s, v(s))| d s \\
& +\sum_{i=1}^{p-1} \frac{\left(t_{p}-t_{i}\right)^{2}}{2 \Gamma(\alpha-2)} \int_{t_{i-1}}^{t_{i}}\left(t_{i}-s\right)^{\alpha-3}|f(s, u(s))-f(s, v(s))| d s \\
& +\sum_{i=1}^{p} \frac{\left(1-t_{p}\right)}{\Gamma(\alpha-1)} \int_{t_{i-1}}^{t_{i}}\left(t_{i}-s\right)^{\alpha-2}|f(s, u(s))-f(s, v(s))| d s \\
& +\sum_{i=1}^{p-1} \frac{\left(1-t_{p}\right)\left(t_{p}-t_{i}\right)}{\Gamma(\alpha-2)} \int_{t_{i-1}}^{t_{i}}\left(t_{i}-s\right)^{\alpha-3}|f(s, u(s))-f(s, v(s))| d s \\
& +\sum_{i=1}^{p} \frac{\left(1-t_{p}\right)^{2}}{2 \Gamma(\alpha-2)} \int_{t_{i-1}}^{t_{i}}\left(t_{i}-s\right)^{\alpha-3}|f(s, u(s))-f(s, v(s))| d s \\
& +\sum_{i=1}^{m-1} \beta\left(t_{m}-t_{i}\right)\left|I_{i}\left(u\left(t_{i}\right)\right)-I_{i}\left(v\left(t_{i}\right)\right)\right|+\sum_{i=1}^{m-1} \frac{\beta\left(t_{m}-t_{i}\right)^{2}}{2}\left|I_{i}^{*}\left(u\left(t_{i}\right)\right)-I_{i}^{*}\left(v\left(t_{i}\right)\right)\right| \\
& +\sum_{i=1}^{m} \beta\left(\eta-t_{m}\right)\left|I_{i}\left(u\left(t_{i}\right)\right)-I_{i}\left(v\left(t_{i}\right)\right)\right|+\sum_{i=1}^{m-1} \beta\left(\eta-t_{m}\right)\left(t_{m}-t_{i}\right)\left|I_{i}^{*}\left(u\left(t_{i}\right)\right)-I_{i}^{*}\left(v\left(t_{i}\right)\right)\right| \\
& +\sum_{i=1}^{m} \frac{\beta\left(\eta-t_{m}\right)^{2}}{2}\left|I_{i}^{*}\left(u\left(t_{i}\right)\right)-I_{i}^{*}\left(v\left(t_{i}\right)\right)\right|+\sum_{i=1}^{p-1}\left(t_{p}-t_{i}\right)\left|I_{i}\left(u\left(t_{i}\right)\right)-I_{i}\left(v\left(t_{i}\right)\right)\right| \\
& +\sum_{i=1}^{p-1} \frac{\left(t_{p}-t_{i}\right)^{2}}{2}\left|I_{i}^{*}\left(u\left(t_{i}\right)\right)-I_{i}^{*}\left(v\left(t_{i}\right)\right)\right|+\sum_{i=1}^{p}\left(1-t_{p}\right)\left|I_{i}\left(u\left(t_{i}\right)\right)-I_{i}\left(v\left(t_{i}\right)\right)\right| \\
& \left.+\sum_{i=1}^{p-1}\left(1-t_{p}\right)\left(t_{p}-t_{i}\right)\left|I_{i}^{*}\left(u\left(t_{i}\right)\right)-I_{i}^{*}\left(v\left(t_{i}\right)\right)\right|+\sum_{i=1}^{p} \frac{\left(1-t_{p}\right)^{2}}{2}\left|I_{i}^{*}\left(u\left(t_{i}\right)\right)-I_{i}^{*}\left(v\left(t_{i}\right)\right)\right|\right\} \\
& \leq \frac{2+\beta\left(1-\eta^{2}\right)}{1-\beta \eta^{2}}\left\{\frac{(p+1) K_{1}}{\Gamma(\alpha+1)}+\frac{(2 p-1) K_{1}}{\Gamma(\alpha)}+\frac{(4 p-3) K_{1}}{2 \Gamma(\alpha-1)}+(2 p-1) K_{2}+\left(2 p-\frac{3}{2}\right) K_{3}\right\} \\
& \times\|u-v\| \\
& \leq \Lambda\|u-v\| .
\end{aligned}
$$


Thus, $\|A u-A v\| \leq \Lambda\|u-v\|$.

As $\Lambda<1$, therefore, $A$ is a contraction. Thus, the conclusion of the theorem follows by the contraction mapping principle.

\section{Examples}

Example 4.1. Consider the following nonlinear impulsive fractional differential equations:

$$
\begin{gathered}
{ }^{C} D^{\alpha} u(t)=\frac{e^{t} \cos ^{3} u(t)}{1+u^{2}(t)}, \quad 0<t<1, \quad t \neq \frac{1}{2} \\
\Delta u^{\prime}\left(\frac{1}{2}\right)=\frac{5+3 u^{4}(1 / 2)}{1+u^{4}(1 / 2)}, \quad \Delta u^{\prime \prime}\left(\frac{1}{2}\right)=3 \sin u\left(\frac{1}{2}\right), \\
u(0)=u^{\prime}(0)=0, \quad \beta u(\eta)=u(1),
\end{gathered}
$$

where $2<\alpha \leq 3,0<\eta<1, \eta \neq 1 / 2,0<\beta<1 / \eta^{2}$, and $p=1$.

Obviously, for $L_{1}=e, L_{2}=5$, and $L_{3}=3$, it is easy to verify condition of Theorem 3.2 holds. Hence, by Theorem 3.2, we can get that the above equation (4.1) has at least one solution.

Example 4.2. Consider the following fractional impulsive the three-point boundary value problem:

$$
\begin{gathered}
{ }^{C} D^{\alpha} u(t)=\frac{e^{5 t} \cos ^{5}\left[3 u(t)+e^{8 u(t)}\right]}{1+u^{4}(t)}+\frac{2 \sin (2 t+5)}{\sqrt{3+u^{2}(t)}}|u(t)|^{\rho}, \quad 0<t<1, \quad t \neq \frac{1}{4}, \\
\Delta u^{\prime}\left(\frac{1}{4}\right)=3+2 \cos ^{2}\left[\ln \left(1+8 u^{2}\left(\frac{1}{4}\right)\right)\right]+3 \cos u\left(\frac{1}{4}\right)\left|u\left(\frac{1}{4}\right)\right|^{\rho 1}, \\
\Delta u^{\prime \prime}\left(\frac{1}{4}\right)=\frac{1}{2}+\frac{5+2 u^{2}(1 / 4)}{2+u^{2}(1 / 4)}+3 e^{-u^{2}(1 / 4)}\left|u\left(\frac{1}{4}\right)\right|^{\rho 2}, \\
u(0)=u^{\prime}(0)=0, \quad \beta u(\eta)=u(1),
\end{gathered}
$$

where $2<\alpha \leq 3,0<\eta<1, \eta \neq 1 / 4,0<\beta<1 / \eta^{2}$, and $p=1$.

Obviously $a=e^{5}, b=2, a_{1}=5, b_{1}=a_{1}^{*}=b_{1}^{*}=3$. It is easy to verify that for $0<$ $\rho, \rho_{k}, \rho_{k}^{*}<1$, the conditions of Theorem 3.3 hold. Therefore, by Theorem 3.3, the impulsive the three-point fractional boundary value problem (4.2) has at least one solution. 
Example 4.3. Consider the following nonlinear impulsive fractional differential equations:

$$
\begin{gathered}
{ }^{C} D^{\alpha} u(t)=t^{2} u^{2}(t)+u(t) \arctan u(t), \quad 0<t<1, \quad t \neq \frac{1}{3} \\
\Delta u^{\prime}\left(\frac{1}{3}\right)=e^{u(t)} u^{3}\left(\frac{1}{3}\right), \quad \Delta u^{\prime \prime}\left(\frac{1}{3}\right)=\frac{3 u^{2}(1 / 3)}{1+2 u^{4}(1 / 3)} \\
u(0)=u^{\prime}(0)=0, \quad \beta u(\eta)=u(1)
\end{gathered}
$$

where $2<\alpha \leq 3,0<\eta<1, \eta \neq 1 / 3,0<\beta<1 / \eta^{2}$, and $p=1$.

Clearly, all the assumptions of Theorem 3.5 hold. Thus, by the conclusion of Theorem 3.5 we can get that (4.3) has at least one solution.

\section{Conclusion}

The existence and uniqueness of solutions to a three-point boundary value problem for fractional nonlinear differential equation with impulses have been discussed. We apply the concepts of fractional calculus together with fixed point theorems to establish the existence results. First of all, we investigate a linear three-point boundary value problem involving fractional derivatives and impulses, which in fact provides the platform to prove the existence of solutions for the associated nonlinear fractional equation with impulses. It is worth mentioning that the conditions of our theorems are easily to verify and that our approach is simple, so they are applicable to a variety of real world problems.

\section{Acknowledgments}

The authors would like to express their gratitude to the anonymous reviewers and editors for their valuable comments and suggestions which improve the quality of present paper. They also would like to thank Professor Bashir Ahmad for his significant suggestions on the improvement of this paper. This paper was supported by the Natural Science Foundation for Young Scientists of Shanxi Province, China (Grant no. 2012021002-3) and Science Foundation of Shanxi Normal University, China (Grant no. ZR1113).

\section{References}

[1] J. Sabatier, O. P. Agrawal, and J. A. T. Machado, Advances in Fractional Calculus: Theoretical Developments and Applications in Physics and Engineering, Dordrecht, The Netherlands, 2007.

[2] I. Podlubny, Fractional Differential Equations, Mathematics in Science and Engineering, Academic Press, New York, NY, USA, 1999.

[3] A. A. Kilbas, H. M. Srivastava, and J. J. Trujillo, Theory and Applications of Fractional Differential Equations, vol. 204 of North-Holland Mathematics Studies, Elsevier Science B.V., Amsterdam, The Netherlands, 2006.

[4] S. G. Samko, A. A. Kilbas, and O. I. Marichev, Fractional Integrals and Derivatives, Theory and Applications, Gordon and Breach, Yverdon, Switzerland, 1993.

[5] V. Lakshmikantham, S. Leela, and J. Vasundhara Devi, Theory of Fractional Dynamic Systems, Cambridge Academic Publishers, Cambridge, UK, 2009. 
[6] J.-H. He, "Approximate analytical solution for seepage flow with fractional derivatives in porous media," Computer Methods in Applied Mechanics and Engineering, vol. 167, no. 1-2, pp. 57-68, 1998.

[7] A. M. A. El-Sayed, "Fractional-order diffusion-wave equation," International Journal of Theoretical Physics, vol. 35, no. 2, pp. 311-322, 1996.

[8] V. Gafiychuk, B. Datsko, and V. Meleshko, "Mathematical modeling of time fractional reaction-diffusion systems," Journal of Computational and Applied Mathematics, vol. 220, no. 1-2, pp. 215-225, 2008.

[9] R. Metzler and J. Klafter, "The restaurant at the end of the random walk: recent developments in the description of anomalous transport by fractional dynamics," Journal of Physics A, vol. 37, no. 31, pp. R161-R208, 2004.

[10] D. Delbosco and L. Rodino, "Existence and uniqueness for a nonlinear fractional differential equation," Journal of Mathematical Analysis and Applications, vol. 204, no. 2, pp. 609-625, 1996.

[11] J. He, "Some applications of nonlinear fractional differential equations and their approximations," Bulletin of Science and Technology, vol. 15, pp. 86-90, 1999.

[12] R. Metzler, W. Schick, H.-G Kilian, and T. F. Nonnenmacher, "Relaxation in filled polymers: a fractional calculus approach," Journal of Chemical Physics, vol. 103, pp. 7180-7186, 1995.

[13] YaE. Ryabov and A. Puzenko, "Damped oscillations in view of the fractional oscillator equation," Physics Review B, vol. 66, article 184201, 2002.

[14] R. P. Agarwal, Y. Zhou, and Y. He, "Existence of fractional neutral functional differential equations," Computers E Mathematics with Applications, vol. 59, no. 3, pp. 1095-1100, 2010.

[15] Y. Zhou and F. Jiao, "Nonlocal Cauchy problem for fractional evolution equations," Nonlinear Analysis, vol. 11, no. 5, pp. 4465-4475, 2010.

[16] J. Wang and Y. Zhou, "A class of fractional evolution equations and optimal controls," Nonlinear Analysis, vol. 12, no. 1, pp. 262-272, 2011.

[17] G.-c. Wu, "A fractional variational iteration method for solving fractional nonlinear differential equations," Computers \& Mathematics with Applications, vol. 61, no. 8, pp. 2186-2190, 2011.

[18] B. Ahmad and J. J. Nieto, "Existence results for a coupled system of nonlinear fractional differential equations with three-point boundary conditions," Computers $\mathcal{E}$ Mathematics with Applications, vol. 58, no. 9, pp. 1838-1843, 2009.

[19] B. Ahmad and S. Sivasundaram, "On four-point nonlocal boundary value problems of nonlinear integro-differential equations of fractional order," Applied Mathematics and Computation, vol. 217, no. 2, pp. 480-487, 2010.

[20] B. Ahmad and A. Alsaedi, "Existence and uniqueness of solutions for coupled systems of higher-order nonlinear fractional differential equations," Fixed Point Theory and Applications, vol. 2010, Article ID 364560, 17 pages, 2010.

[21] J. J. Nieto, "Maximum principles for fractional differential equations derived from Mittag-Leffler functions," Applied Mathematics Letters, vol. 23, no. 10, pp. 1248-1251, 2010.

[22] S. Zhang, "Positive solutions to singular boundary value problem for nonlinear fractional differential equation," Computers \& Mathematics with Applications, vol. 59, no. 3, pp. 1300-1309, 2010.

[23] K. Balachandran and J. J. Trujillo, "The nonlocal Cauchy problem for nonlinear fractional integrodifferential equations in Banach spaces," Nonlinear Analysis A, vol. 72, no. 12, pp. 4587-4593, 2010.

[24] D. Baleanu and J. J. Trujillo, "On exact solutions of a class of fractional Euler-Lagrange equations," Nonlinear Dynamics, vol. 52, no. 4, pp. 331-335, 2008.

[25] G. Wang, S. K. Ntouyas, and L. Zhang, "Positive solutions of the three-point boundary value problem for fractional-order differential equations with an advanced argument," Advances in Difference Equations, vol. 2011, p. 2, 2011.

[26] A. Cabada and G. Wang, "Positive solutions of nonlinear fractional differential equations with integral boundary value conditions," Journal of Mathematical Analysis and Applications, vol. 389, pp. 403-411, 2012.

[27] G. Wang and L. Zhang, "Iterative approximation of solution for nonlinear fractional differential equation with nonseparated integral boundary conditions," Nonlinear Analysis. In press.

[28] G. Wang, "Monotone iterative technique for boundary value problems of nonlinear fractional differential equation with deviating arguments," Journal of Computational and Applied Mathematics, vol. 236, pp. 2425-2430, 2012.

[29] G. Wang, R. P. Agarwal, and A. Cabada, "Existence results and monotone iterative technique for systems of nonlinear fractional differential equations," Applied Mathematics Letters, vol. 25, pp. 1019 1024, 2012. 
[30] L. Zhang, G. Wang, and G. Song, "On mixed boundary value problem of impulsive semilinear evolution equations of fractional order," Boundary Value Problems, vol. 2012, p. 17, 2012.

[31] L. Zhang and G. Wang, "Existence of solutions for nonlinear fractional differential equations with impulses and anti-periodic boundary conditions," Electronic Journal of Qualitative Theory of Differential Equations, vol. 7, pp. 1-11, 2011.

[32] G. Wang, B. Ahmad, and L. Zhang, "Some existence results for impulsive nonlinear fractional differential equations with mixed boundary conditions," Computers \& Mathematics with Applications, vol. 62, no. 3, pp. 1389-1397, 2011.

[33] G. Wang, B. Ahmad, and L. Zhang, “Impulsive anti-periodic boundary value problem for nonlinear differential equations of fractional order," Nonlinear Analysis, vol. 74, no. 3, pp. 792-804, 2011.

[34] B. Ahmad and G. Wang, "A study of an impulsive four-point nonlocal boundary value problem of nonlinear fractional differential equations," Computers \& Mathematics with Applications, vol. 62, no. 3, pp. 1341-1349, 2011.

[35] B. Ahmad and J. J. Nieto, “Existence of solutions for impulsive anti-periodic boundary value problems of fractional order," Taiwanese Journal of Mathematics, vol. 15, no. 3, pp. 981-993, 2011.

[36] B. Ahmad and S. Sivasundaram, "Existence results for nonlinear impulsive hybrid boundary value problems involving fractional differential equations," Nonlinear Analysis, vol. 3, no. 3, pp. 251-258, 2009.

[37] B. Ahmad and S. Sivasundaram, "Existence of solutions for impulsive integral boundary value problems of fractional order," Nonlinear Analysis, vol. 4, no. 1, pp. 134-141, 2010.

[38] R. P. Agarwal and B. Ahmad, "Existence of solutions for impulsive anti-periodic boundary value problems of fractional semilinear evolution equations," Dynamics of Continuous, Discrete $\mathcal{E}$ Impulsive Systems A, vol. 18, no. 4, pp. 457-470, 2011.

[39] G. M. Mophou, "Existence and uniqueness of mild solutions to impulsive fractional differential equations," Nonlinear Analysis A, vol. 72, no. 3-4, pp. 1604-1615, 2010.

[40] J. Henderson and A. Ouahab, "Impulsive differential inclusions with fractional order," Computers $\mathcal{E}$ Mathematics with Applications, vol. 59, no. 3, pp. 1191-1226, 2010.

[41] Y. Tian and Z. Bai, "Existence results for the three-point impulsive boundary value problem involving fractional differential equations," Computers $\mathcal{E}$ Mathematics with Applications, vol. 59, no. 8, pp. 26012609, 2010.

[42] X. Zhang, X. Huang, and Z. Liu, "The existence and uniqueness of mild solutions for impulsive fractional equations with nonlocal conditions and infinite delay," Nonlinear Analysis, vol. 4, no. 4, pp. 775-781, 2010.

[43] A. V. Bicadze and A. A. Samarskiı̌, "Some elementary generalizations of linear elliptic boundary value problems," Doklady Akademii Nauk SSSR, vol. 185, pp. 739-740, 1969.

[44] V. A. Il'in and E. I. Moiseev, "Nonlocal boundary-value problem of the first kind for a Sturm-Liouville operator in its differential and finite difference aspects," Journal of Differential Equations, vol. 23, no. 7, pp. 803-810, 1987.

[45] P. W. Eloe and B. Ahmad, "Positive solutions of a nonlinear $n$th order boundary value problem with nonlocal conditions," Applied Mathematics Letters, vol. 18, no. 5, pp. 521-527, 2005.

[46] G. Infante and J. R. L. Webb, "Nonlinear non-local boundary-value problems and perturbed Hammerstein integral equations," Proceedings of the Edinburgh Mathematical Society, vol. 49, no. 3, pp. 637656, 2006.

[47] Z. Du, X. Lin, and W. Ge, "Nonlocal boundary value problem of higher order ordinary differential equations at resonance," The Rocky Mountain Journal of Mathematics, vol. 36, no. 5, pp. 1471-1486, 2006.

[48] J. R. Graef and B. Yang, "Positive solutions of a third order nonlocal boundary value problem," Discrete and Continuous Dynamical Systems S, vol. 1, no. 1, pp. 89-97, 2008.

[49] J. R. L. Webb, “Nonlocal conjugate type boundary value problems of higher order," Nonlinear Analysis A, vol. 71, no. 5-6, pp. 1933-1940, 2009.

[50] C. P. Gupta, "Solvability of a three-point nonlinear boundary value problem for a second order ordinary differential equation," Journal of Mathematical Analysis and Applications, vol. 168, no. 2, pp. 540$551,1992$.

[51] M. K. Kwong and J. S. W. Wong, "Solvability of second-order nonlinear three-point boundary value problems," Nonlinear Analysis A, vol. 73, no. 8, pp. 2343-2352, 2010.

[52] J. X. Sun, Nonlinear Functional Analysis and its Application, Science Press, Beijing, China, 2008. 


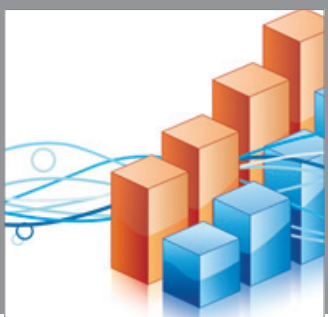

Advances in

Operations Research

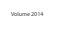

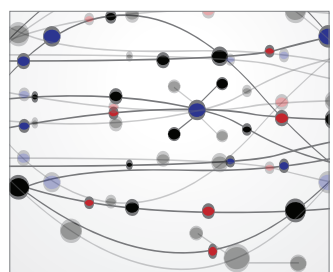

\section{The Scientific} World Journal
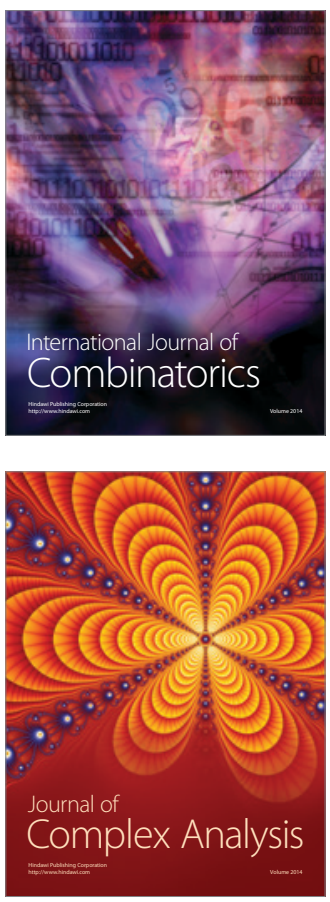

International Journal of

Mathematics and

Mathematical

Sciences
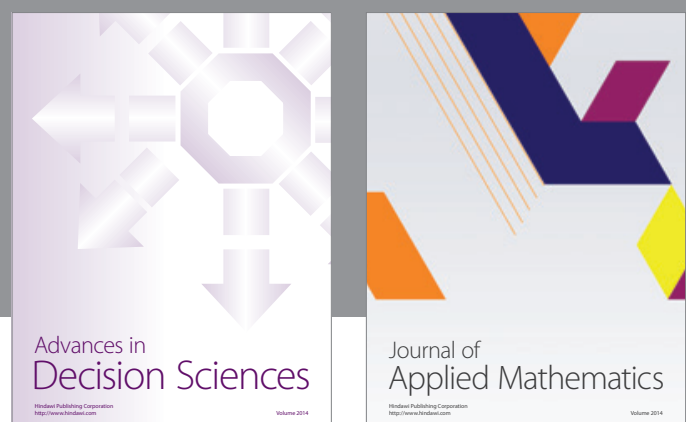

Journal of

Applied Mathematics
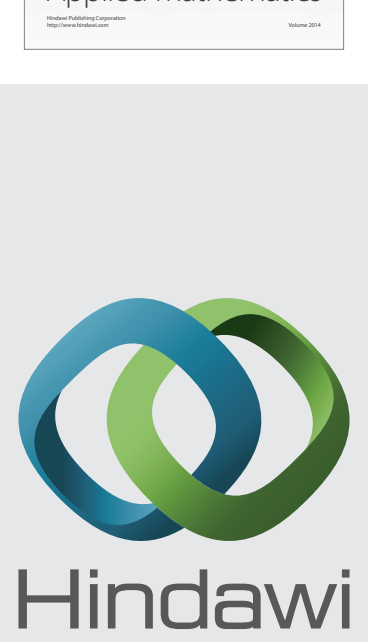

Submit your manuscripts at http://www.hindawi.com
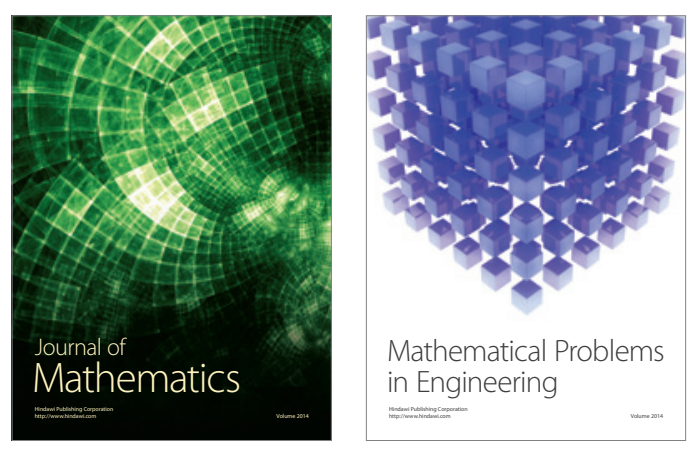

Mathematical Problems in Engineering
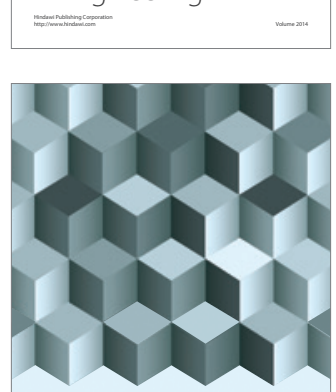

Journal of

Function Spaces
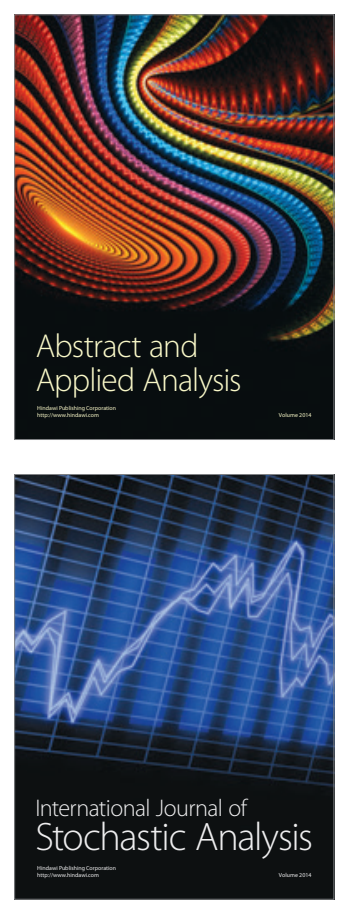

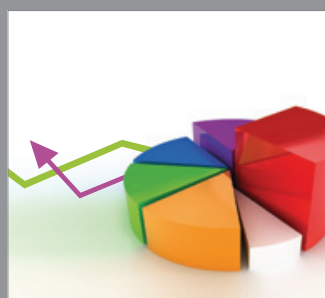

ournal of

Probability and Statistics

Promensencen
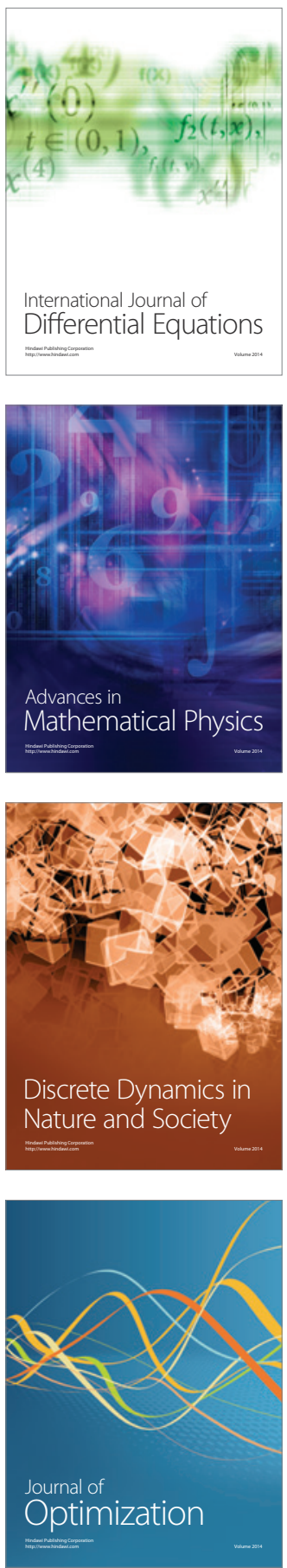